\title{
Holling-Tanner Predator-Prey Model with State-Dependent Feedback Control
}

\author{
Jin Yang $\mathbb{D},{ }^{1}$ Guangyao Tang, ${ }^{2}$ and Sanyi Tang $\mathbb{D}^{3}$ \\ ${ }^{1}$ Department of Mathematics, Chongqing Jiaotong University, Chongqing 400074, China \\ ${ }^{2}$ Key Laboratory of Biologic Resources Protection and Utilization, Hubei Minzu University, Enshi, Hubei 445000, China \\ ${ }^{3}$ College of Mathematics and Information Science, Shaanxi Normal University, Xian, 710062, China
}

Correspondence should be addressed to Jin Yang; seehom@126.com

Received 17 April 2018; Accepted 3 October 2018; Published 18 October 2018

Academic Editor: Zhengqiu Zhang

Copyright (C) 2018 Jin Yang et al. This is an open access article distributed under the Creative Commons Attribution License, which permits unrestricted use, distribution, and reproduction in any medium, provided the original work is properly cited.

\begin{abstract}
In this paper, we propose a novel Holling-Tanner model with impulsive control and then provide a detailed qualitative analysis by using theories of impulsive dynamical systems. The Poincaré map is first constructed based on the phase portraits of the model. Then the main properties of the Poincaré map are investigated in detail which play important roles in the proofs of the existence of limit cycles, and it is concluded that the definition domain of the Poincaré map has a complicated shape with discontinuity points under certain conditions. Subsequently, the existence of the boundary order-1 limit cycle is discussed and it is shown that this limit cycle is unstable. Furthermore, the conditions for the existence and stability of an order-1 limit cycle are provided, and the existence of order- $k(k \geq 2)$ limit cycle is also studied. Moreover, numerical simulations are carried out to substantiate our results. Finally, biological implications related to the mathematical results which are beneficial for successful pest control are addressed in the Conclusions section.
\end{abstract}

\section{Introduction}

Since Lotka-Volterra systems have made great efforts on the mathematical models of predator-prey interactions, many studies were carried out to develop predator-prey systems with the aim of solving problems originating from real world phenomena. In particular, one of the most important and famous biological models named the Holling-Tanner model or known as the model of R. M. May has become a hot topic and has been studied by many scholars [1-7]; it can be described by the following differential equations:

$$
\begin{aligned}
& \frac{d x(t)}{d t}=r x(t)\left(1-\frac{x(t)}{K}\right)-\frac{m x(t) y(t)}{A+x(t)}, \\
& \frac{d y(t)}{d t}=s y(t)\left(1-\frac{y(t)}{h x(t)}\right),
\end{aligned}
$$

where $x(t), y(t)$ are the densities of the prey and predator populations at time $t$, respectively. $r$ represents the intrinsic growth rate of the prey, $K$ represents the carrying capacity of the prey, $m$ is the maximal predator per capita consumption rate, that is, the maximum number of preys that can be eaten by a predator in each time unit, $A$ is the number of preys necessary to achieve one-half of the maximum rate $m, s$ represents the intrinsic growth rate of the predator, and $h$ is a measure of the food quality that the prey provides for conversion into predator births. Note that the dynamics of system (1) have been investigated by many scholars [1-7].

Naturally, the $x(t)$ of system (1) usually denotes the pests population; integrated pest management (IPM) is needed to be implemented in order to control the pest population within a safe range [8-10], where IPM includes biological control, chemical control, or their combinations. Furthermore, biological control often consists of releasing enemies, harvesting, and catching, etc., whilst chemical control involves spraying pesticides [11, 12].

To investigate the global dynamics of the predator-prey systems concerning IPM and to further explore how IPM affects the corresponding successful control strategies, the predator-prey systems with impulsive control strategy are commonly proposed to model the IPM with releasing natural enemies and spraying pesticide at different fixed periods 
[11-13]. In these studies, the permanence, the stability of the pest-free periodic solution, and the conditions for the coexistence of pest and natural enemies are addressed. Although the applications of IPM at fixed times can achieve the purpose of pest control, many negative effects have been detected. For example, the overuse of pesticides has resulted in the enhancement of drug resistance, environmental pollution, and cost increases, etc.

In practice, state-dependent feedback control is proved to be more reasonable to depict problems originating from real world phenomena than fixed time pulses [14], and it is often described by using impulsive dynamical systems, which have received a lot of attention [14-21], and revealing that the control tactics should only be applied once the states of the model reach a prescribed given threshold. However, none of the authors expanded the system (1) to include the effects of state-dependent feedback control with IPM owing to the complexity of the system (1). Therefore, the main subjects are to investigate the model (1) with effects of IPM by considering the impulsive strategy; these modifications derive the following model:

$$
\begin{aligned}
& \frac{d x(t)}{d t}=r x(t)\left(1-\frac{x(t)}{K}\right)-\frac{m x(t) y(t)}{A+x(t)}, \\
& \frac{d y(t)}{d t}=s y(t)\left(1-\frac{y(t)}{h x(t)}\right),
\end{aligned}
$$$$
x(t)<E T,
$$

$$
\begin{aligned}
& x\left(t^{+}\right)=(1-\delta) x(t), \\
& y\left(t^{+}\right)=(1+q) y(t)+\sigma,
\end{aligned}
$$

$$
x(t)=E T,
$$

where $\delta>0$ represents the fatality rate for the prey due to chemical control, $q>0$ and $\sigma>0$ denotes the release number of $y(t)$. Denote $x\left(0^{+}\right)$and $y\left(0^{+}\right)$as the initial densities of $x(t)$ and $y(t)$. In this paper we assume that $x\left(0^{+}\right)$is always less than ET for biological implications. When the number of preys reaches $E T$ at time $t$, then control strategies are initiated and the number of $x(t)$ and $y(t)$ becomes $(1-\delta) E T$ and $(1+q) y(t)+\sigma$, respectively. Note that a more general case of system (2) has been studied by Nie and coauthors without concerning the dynamics of system (1) [22]. In this paper, we will present novel analytical methods to study system (2) based on the dynamics of system (1); we will not only provide exact domains of the phase sets and impulsive sets when system (1) exhibits different dynamical behaviour, but also discuss the main properties of the Poincaré map, in addition to the existence of an order-3 limit cycle, which are different from reference [22].

The paper is arranged as follows: we introduce many important definitions and lemmas of the planar impulsive dynamical systems in Section 2. In Section 3, we first construct the Poincaré map and then the complex properties of the Poincaré map are discussed. Further the conditions which guarantee the existence of the boundary order-1 limit cycle are obtained, and then it is concluded that this limit cycle is unstable. Subsequently, the existence and stability of an order-1 limit cycle will be addressed, and the existence of order- $k(k \geq 2)$ limit cycles is also studied. In Section 4 , the complex domains of impulsive set and phase set are provided for system (2) and many interesting results are indicated. Moreover, numerical studies are employed not only to verify the results but also to reveal the complexity of system (2). Finally, some biological implications of the results are discussed and some conclusions are presented.

\section{Preliminaries and Main Properties of System (1)}

The generalized planar impulsive semidynamical systems with control are usually described by

$$
\begin{aligned}
& \frac{d x(t)}{d t}=P(x, y), \\
& \frac{d y(t)}{d t}=Q(x, y), \\
& x^{+}=x+\alpha(x, y), \\
& y^{+}=y+\beta(x, y) \notin \mathscr{I}, \\
& \quad(x, y) \in \mathscr{I},
\end{aligned}
$$

where $(x, y) \in R^{2}$; we denote $x^{+}=x\left(t^{+}\right)$and $y^{+}=y\left(t^{+}\right)$ for simplicity, and $P, Q, \alpha, \beta$ are continuous functions from $R^{2}$ into $R ; \mathscr{I} \subset R^{2}$ represents the impulsive set. For each point $z(x, y) \in \mathscr{I}$, the map $I: R^{2} \longrightarrow R^{2}$ is defined via

$$
\begin{aligned}
z^{+} & =I(z)=(x+\alpha(x, y), y+\beta(x, y))=\left(x^{+}, y^{+}\right) \\
& \in R^{2}
\end{aligned}
$$

and $z^{+}$is called an impulsive point of $z$.

Let $\mathscr{P}=I(\mathscr{I})$ be the phase set (that is, for any $z \in$ $\left.\mathscr{I}, I(z)=z^{+} \in \mathscr{P}\right)$, and $\mathscr{P} \cap \mathscr{I}=\varnothing$. In the following some definitions related to impulsive semidynamical systems will be listed briefly, which are used in this work.

Assume $(X, \Pi, R)$ or $(X, \Pi)$ to be impulsive dynamical system $[23,24], X$ denotes a metric space, and $R_{+}$denotes a set of positive reals. For arbitrary $z \in X$, assume $\Pi_{z}(t)=$ $\Pi(z, t)$ defined as $\Pi_{z}: R \longrightarrow X$ which satisfies $\Pi(z, 0)=z$ for arbitrary $z \in X$, and $\Pi(\Pi(z, t), s)=\Pi(z, t+s)$ for arbitrary $z \in X$ and $t, s \in R_{+}$. Let $G(z)=\bigcup\{G(z, t) \mid t \in R\}$ and $G(z, t)=\{w \in X \mid \Pi(w, t)=z\}$ be the attainable set of $z$ at $t \in R_{+}$. Besides, denote $\mathscr{I}(z)=\mathscr{I}^{+}(z) \cup \mathscr{I}^{-}(z)$. The following Definitions and Lemmas are also important for discussing the dynamics of system (2) [25-30].

Definition 1. An impulsive semidynamical system $(X, \Pi$; $\mathscr{I}, I)$ consists of a continuous semidynamical system $(X, \Pi)$ together with a nonempty closed subset $\mathscr{I}$ (or impulsive set) of $X$ and a continuous function $I: \mathscr{I} \longrightarrow X$ such that the following property holds: no point $z \in X$ is a limit point of $\mathscr{I}(z) ;\{t \mid G(z, t) \cap \mathscr{I} \neq \varnothing\}$ is a closed subset of $R$. 
We denote the points of discontinuity of $\Pi_{z}$ by $\left\{z_{n}^{+}\right\}$, and we define a function $\Phi$ from $X$ into the extended positive reals $R \cup\{\infty\}$ as follows: let $z \in X$; if $\mathscr{I}^{+}(z)=\varnothing$ we set $\Phi(z)=\infty$; otherwise $\mathscr{I}^{+}(z) \neq \varnothing$ and we set $\Phi(z)=s$, where $\Pi(x, t) \notin \mathscr{I}$ for $0<t<s$ but $\Pi(z, s) \in \mathscr{I}$.

Definition 2. A trajectory $\Pi_{z}$ in $(X, \Pi, \mathscr{F}, I)$ is said to be periodic of period $T_{k}$ and order $k$ if there exist nonnegative integers $m \geq 0$ and $k \geq 1$ such that $k$ is the smallest integer for which $z_{m}^{+}=z_{m+k}^{+}$and $T_{k}=\sum_{i=m}^{m+k-1} \Phi\left(z_{i}\right)=\sum_{i=m}^{m+k-1} s_{i}$.

Lemma 3 (see $[23,24])$. The T-periodic solution $(x, y)=$ $(\xi(t), \eta(t))$ of system

$$
\begin{aligned}
& \frac{d x}{d t}=P(x, y), \\
& \frac{d y}{d t}=Q(x, y),
\end{aligned}
$$$$
\text { if } \phi(x, y) \neq 0 \text {, }
$$

$$
\begin{aligned}
& x^{+}=x+\alpha(x, y), \\
& y^{+}=y+\beta(x, y),
\end{aligned}
$$$$
\text { if } \phi(x, y)=0 \text {, }
$$

is orbitally asymptotically stable and enjoys the property of asymptotic phase if the Floquet multiplier $\mu_{2}$ satisfies the condition $\left|\mu_{2}\right|<1$, where

$$
\begin{aligned}
\mu_{2} & =\prod_{k=1}^{q} \Delta_{k} \\
& \cdot \exp \left(\int_{0}^{T}\left[\frac{\partial P}{\partial x}(\xi(t), \eta(t))+\frac{\partial Q}{\partial y}(\xi(t), \eta(t))\right] d t\right),
\end{aligned}
$$

with

$$
\Delta_{k}=\frac{P_{+}((\partial \beta / \partial y)(\partial \phi / \partial x)-(\partial \beta / \partial x)(\partial \phi / \partial y)+(\partial \phi / \partial x))+Q_{+}((\partial \alpha / \partial x)(\partial \phi / \partial y)-(\partial \alpha / \partial y)(\partial \phi / \partial x)+(\partial \phi / \partial y))}{P(\partial \phi / \partial x)+Q(\partial \phi / \partial y)},
$$

and $\phi(x, y)$ is in $C^{1}$. We denote $(x, y) \notin \mathscr{I}$ by $\phi(x, y) \neq 0$. $P, Q, \partial \alpha / \partial x, \partial \alpha / \partial y, \partial \beta / \partial x, \partial \beta / \partial y, \partial \phi / \partial x$ and $\partial \phi / \partial y$ can be calculated at $\left(\xi\left(t_{k}\right), \eta\left(t_{k}\right)\right)$; let $P_{+}=P\left(\xi\left(t_{k}^{+}\right), \eta\left(t_{k}^{+}\right)\right)$and $Q_{+}=Q\left(\xi\left(t_{k}^{+}\right), \eta\left(t_{k}^{+}\right)\right) . t_{k}(k, q \in N, N$ is positive integers $)$ is $k$-th jump time.

Noting that many studies about system (1) can be found [1-7], based on these studies, we let $L_{1}$ and $L_{2}$ be two isoclines of system (1):

$$
\begin{aligned}
& L_{1}: y=h x, \\
& L_{2}: y=\frac{r}{m}\left(1-\frac{x}{K}\right)(A+x) .
\end{aligned}
$$

There are two equilibria in system $(1)$, that is, $(K, 0)$ and the unique interior equilibrium $E^{*}\left(x^{*}, y^{*}\right)$ with $y^{*}=h x^{*}$, and with $\Lambda=r K-h m K-r A$

$$
x^{*}=\frac{\Lambda+\sqrt{(-\Lambda)^{2}+4 r^{2} K A}}{2 r} .
$$

Define the function

$$
f(x)=2 x^{2}+\left(\frac{A}{K}+\frac{s}{r}-1\right) x+\frac{A s}{K r},
$$

and then denote the two positive roots of the function $f(x)$ as $\alpha_{1}$ and $\alpha_{2}$ (assume that $\alpha_{1}<\alpha_{2}$ ) if they exist. Based on the discussions in [1-7], we get the following result.

Lemma 4. (i) If $f(x) \geq 0$, then $E^{*}$ is globally asymptotically stable in the interior of the first quadrant.

(ii) If $f(x)<0$ and $\alpha_{1}<x^{*}<\alpha_{2}$, then $E^{*}$ is unstable and system (1) exists with a stable limit cycle. (iii) Under certain conditions (for example, $0<x^{*}<\alpha_{1}$ and $V_{1}>0$, for details see $[3,7]$ ), then $E^{*}$ is locally stable and system (1) has two limit cycles with the outermost being stable and the innermost being unstable.

In the light of the above Definitions and Lemmas, we next focus on the constuctions of the Poincaré map and the global dynamical behaviours of system (2).

\section{Poincaré Map and Order $-k$ Limit Cycle}

In order to study the dynamics of system (2), the Poincaré map which is determined by the impulsive points in the phase set needs to be constructed first.

Define two lines as follows:

$$
\begin{aligned}
& L_{3}: x=(1-\delta) E T, \\
& L_{4}: x=E T .
\end{aligned}
$$

Since $0<E T<K$, substituting $x=E T$ into the line $L_{2}$, one yields the intersection point of $L_{2}$ and $L_{4}$, denoted as $Q\left(E T, y_{E}\right)$ with

$$
y_{E}=\frac{r}{m}\left(1-\frac{E T}{K}\right)(A+E T) .
$$

Then denote the intersection point of $L_{2}$ and $L_{3}$ as $P((1-$ $\left.\delta) E T, y_{\delta E}\right)$ with

$$
y_{\delta E}=\frac{r}{m}\left(1-\frac{(1-\delta) E T}{K}\right)(A+(1-\delta) E T) .
$$

Denote the intersection point of $L_{1}$ and $L_{3}$ as $R((1-$ $\delta) E T, y_{\delta E}^{*}$ ) with $y_{\delta E}^{*}=h(1-\delta) E T$. 
To define the impulsive semidynamical system for model (2), the exact domains of impulsive sets and phase sets should be addressed. To this end, based on the positions between the threshold $E T$ and the equilibrium $E^{*}$, we consider the following two cases:

$$
\begin{aligned}
& \left(A_{1}\right) \quad E T<x^{*}, \\
& \left(A_{2}\right) \quad E T \geq x^{*} .
\end{aligned}
$$

For case $\left(A_{1}\right), L_{3}$ and $L_{4}$ are both located to the left of the equilibrium $E^{*}$. According to the vector fields of the model (2), any solution initiating from the line $L_{3}$ will reach the line $L_{4}$ in a finite time. Then the impulsive set $\mathscr{I}$ for system (2) can be determined as follows:

$$
\mathscr{I}=\left\{(x, y) \in R^{2} \mid x=E T, 0 \leq y \leq y_{E},\right\}
$$

which is a closed subset of $R^{2}$. Moreover, define the continuous function $I$ as follows:

$$
\begin{aligned}
I:(E T, y) & \in \mathscr{I} \longrightarrow\left(x^{+}, y^{+}\right) \\
& =((1-\delta) E T,(1+q) y+\sigma) \in R^{2} .
\end{aligned}
$$

So the phase set $\mathscr{P}$ can be defined, where

$$
\begin{aligned}
\mathscr{P} & =I(\mathscr{I}) \\
& =\left\{\left(x^{+}, y^{+}\right) \in R^{2} \mid x^{+}=(1-\delta) E T, y^{+} \in D_{0}\right\}
\end{aligned}
$$

with $D_{0}=\left[\sigma,(1+q) y_{E}+\sigma\right]$. Therefore, based on the above analysis model (2) defines an impulsive semidynamical system $(X, \Pi ; \mathscr{J}, I)$.

For simplicity, assume that $\left(x_{0}^{+}, y_{0}^{+}\right) \in \mathscr{P}$. We use $D_{0}=$ $[\sigma,+\infty)$ to define the Poincaré map because any trajectory of system (2) with initial condition $y_{0}^{+}>(1+q) y_{E}+\sigma$ will experience one time impulsive effect and then satisfy $y_{k}^{+} \leq$ $(1+q) y_{E}+\sigma$ for all $k \geq 1$.

For case $\left(A_{2}\right), L_{4}$ is located to the right of the equilibrium $E^{*}$, while the locations of $L_{3}$ could lie on the left (or right) of the equilibrium $E^{*}$. According to Lemma 4, system (2) could possess a global stable equilibrium $E^{*}$, or unique stable limit cycle $\Omega$, or two limit cycles with the outermost $\Omega_{2}$ being stable and the innermost $\Omega_{1}$ being unstable under different set of conditions. Thus, any solution initiating from $\left((1-\delta) E T, y_{0}^{+}\right)$with $y_{0}^{+}>0$ will experience infinitely many pulses or will be free from impulsive effects, depending on the initial conditions. For example, for case (i) of Lemma 4, there exists a curve $\Lambda_{2}$ which is tangential to the line $L_{4}$ at a point $Q\left(E T, y_{E}\right)$, and the curve $\Lambda_{2}$ must intersect the line $L_{2}$ at a point $P_{1}\left(x_{P_{1}}, y_{P_{1}}\right)$ such that $\Lambda_{2}$ is tangential to the line $L_{2}$ at this point. If $\left(1-p_{0}\right) E T<x_{P_{1}}$, then any solution initiating from $\left((1-\delta) E T, y_{0}^{+}\right)$with $y_{0}^{+} \in D_{0}$ experiences infinitely many pulses. If $\left(1-p_{0}\right) E T \geq x_{P_{1}}$, then the curve $\Lambda_{2}$ must intersect the line $L_{3}$ at two points, denoted by $T_{1}=$ $\left(\left(1-p_{0}\right) E T, y_{\text {min }}^{P}\right)$ and $T_{2}=\left(\left(1-p_{0}\right) E T, y_{\text {max }}^{P}\right)$. Moreover, any solutions initiating from $\left(x_{0}^{+}, y_{0}^{+}\right)$with $y_{\text {min }}^{P}<y_{0}^{+}<y_{\text {max }}^{P}$ will be free from impulsive effects. Therefore, the exact domains of impulsive sets and phase sets of system (2) could vary which will lead to complex dynamical behavior for system (2) under case $\left(A_{2}\right)$; those will be discussed after investigations for case $\left(A_{1}\right)$.
3.1. Poincaré Map for Case $\left(A_{1}\right)$. To define the Poincaré map for system (2), denote two sections as follows:

$$
\begin{aligned}
& S_{0}=\{(x, y) \mid x=(1-\delta) E T, y \geq 0\}, \\
& S_{1}=\{(x, y) \mid x=E T, y \geq 0\} .
\end{aligned}
$$

We choose section $S_{0}$ as a Poincaré section. Assume that the point $H_{k}^{+}\left((1-\delta) E T, y_{k}^{+}\right)$lies in the section $S_{0}$, and the trajectory initiating from point $H_{k}^{+}$intersects the section $S_{1}$ at the point $H_{k+1}\left(E T, y_{k+1}\right)$ in a finite time, where $y_{k+1}$ is only determined by $y_{k}^{+}$; that is $y_{k+1}=g\left(y_{k}^{+}\right)$. Further, point $H_{k+1}$ experiences one time impulsive effect and then maps to the point $H_{k+1}^{+}\left((1-\delta) E T, y_{k+1}^{+}\right)$which lies on the section $S_{0}$, where $y_{k+1}^{+}=(1+q) y_{k+1}+\sigma$. Therefore, the Poincaré map with respect to impulsive point series of system (2) can be defined as

$$
y_{i+1}^{+}=(1+q) g\left(y_{i}^{+}\right)+\sigma \triangleq \varphi\left(y_{i}^{+}\right) .
$$

To investigate the existence of periodic solutions for system (2), we define the Poincaré map $\varphi$ in the phase set according to the phase portrait of model (1). Thus, we denote

$$
\begin{aligned}
& P(x(t), y(t))=r x(t)\left(1-\frac{x(t)}{K}\right)-\frac{m x(t) y(t)}{A+x(t)}, \\
& Q(x(t), y(t))=s y(t)\left(1-\frac{y(t)}{h x(t)}\right),
\end{aligned}
$$

leading to the following scalar differential equation in phase space

$$
\begin{aligned}
\frac{d y}{d x} & =\frac{s y(1-y / h x)}{r x(1-x / K)-m x y /(A+x)} \\
& \doteq \omega(x, y), \\
y((1-\delta) E T) & =y_{0}^{+} .
\end{aligned}
$$

For model (21), we only focus on the region $\Omega$, where

$$
\begin{aligned}
\Omega & =\{(x, y) \mid x>0, y>0, y \\
& \left.<\frac{r}{m}\left(1-\frac{x}{K}\right)(A+x)\right\},
\end{aligned}
$$

and in this region the function $\omega(x, y)$ is continuously differentiable. Denote $x_{0}^{+}=(1-\delta) E T, y_{0}^{+} \doteq S, S \in \mathscr{P}$ with $S<y_{\delta E}$; that is to say $\left(x_{0}^{+}, y_{0}^{+}\right) \in \Omega$. Then we get

$$
\begin{aligned}
y(x)=y(x ;(1-\delta) E T, S)=y(x, S), & \\
& (1-\delta) E T \leq x \leq E T,
\end{aligned}
$$

and it follows from model (21) that

$$
y(x, S)=S+\int_{(1-\delta) E T}^{x} \omega(s, y(s, S)) d s .
$$

Then the Poincaré map $\varphi$ related to the phase portrait of model (1) takes the following form:

$$
\varphi(S)=(1+q) y(E T, S)+\sigma .
$$




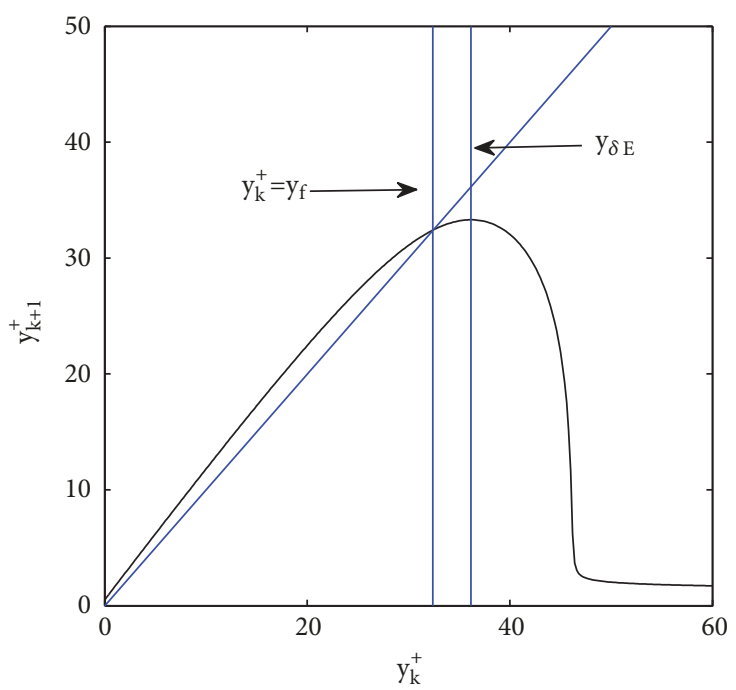

(a)

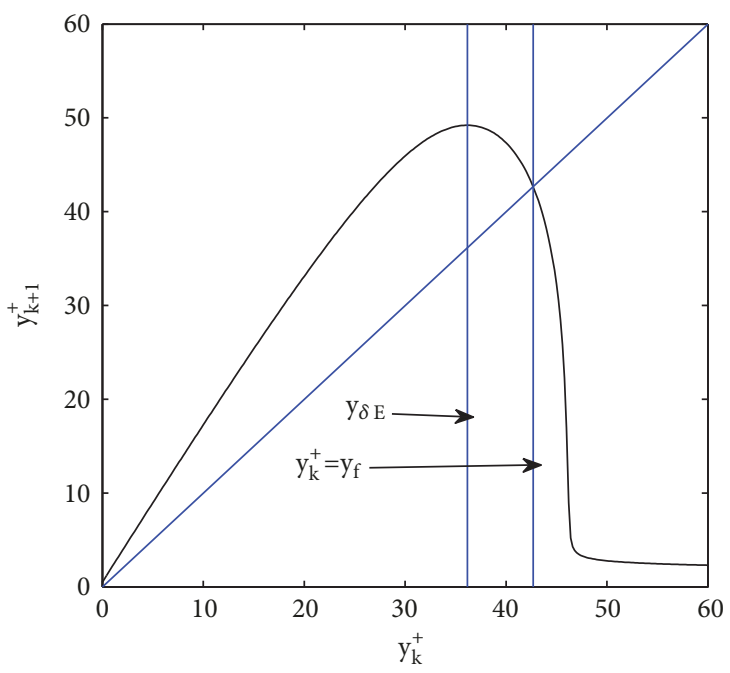

(c)

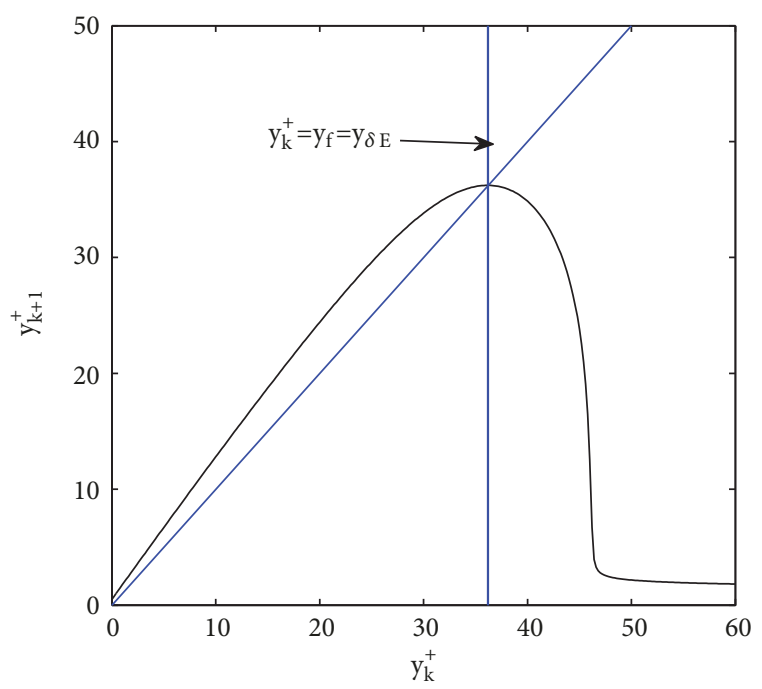

(b)

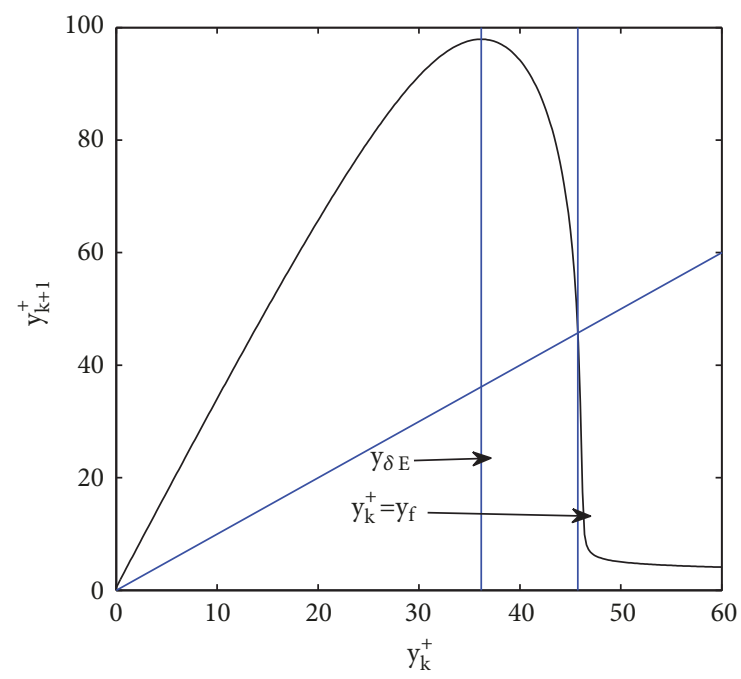

(d)

Figure 1: The Poincaré map $\varphi$ and its fixed point $y_{f}$. All parameter values were fixed as $r=0.5, K=50, m=0.15, A=0.5, s=0.05, h=0.7$, $E T=30, \delta=0.5$, and $\sigma=0.5$. (a) $q=0.01$; (b) $q=0.1$; (c) $q=0.5$; (d) $q=2$.

Since the formation of the Poincaré map $\varphi$ has been investigated, it is possible to address the corresponding properties which are useful in the rest of the paper.

Theorem 5. For case $\left(A_{1}\right)$, the Poincaré map $\varphi$ of system (2) satisfies the following properties, as shown in Figure 1:

(I) The domain and range of $\varphi$ are $[0,+\infty)$ and $\left[\sigma, \varphi\left(y_{\delta E}\right)\right)=\left[\sigma,(1+q) y\left((1-\delta) E T, y_{\delta E}\right)+\sigma\right]$, respectively. It is increasing on $\left[0, y_{\delta E}\right]$ and decreasing on $\left[y_{\delta E},+\infty\right)$.

(II) For all $y<y_{\delta E}^{*}$, the inequality $\varphi(y) \geq(1+q) y+\sigma \geq y$ holds true.

(III) $\varphi$ is continuously differentiable.

(IV) $\varphi$ is concave on $\left[y_{\delta E}^{*}, y_{\delta E}\right)$.

(V) $\varphi$ has a unique positive fixed point $y_{f}$.

(VI) $\varphi$ has a horizontal asymptote $y=\sigma$ as $y_{k}^{+} \longrightarrow+\infty$.
Proof. (I) According to the vector field of system (1), the domain of $\varphi$ is defined on the interval $[0, \infty)$. In phase set, for any $y_{i}^{+}, y_{j}^{+} \in\left[0, y_{\delta E}\right]$ with $y_{i}^{+}<y_{j}^{+}$, we have $y_{i+1}<y_{j+1}$ due to the Cauchy-Lipschitz Theorem. In fact, according to the vector fields of system (2) we have $d y / d t<0$ and $d x / d t>0$ for $y \in\left[y_{\delta E}^{*}, y_{\delta E}\right]$, and $d y / d t>0$ and $d x / d t>0$ in the region below $L_{1}$. Thereby, the solutions starting from $\left((1-\delta) E T, y_{i}^{+}\right)$ and $\left((1-\delta) E T, y_{j}^{+}\right)$will first cross $L_{1}$ and then meet $x=E T$ at points $\left((1-\delta) E T, y_{i+1}\right)$ and $\left((1-\delta) E T, y_{j+1}\right)$ with $y_{i+1}<y_{j+1}$. If $y \in\left[0, y_{\delta E}^{*}\right)$, then $d y / d t>0$ and $d x / d t>0$, so the solutions starting from $\left((1-\delta) E T, y_{i}^{+}\right)$and $\left((1-\delta) E T, y_{j}^{+}\right)$will meet at $x=E T$ at points $\left((1-\delta) E T, y_{i+1}\right)$ and $\left((1-\delta) E T, y_{j+1}\right)$ with $y_{i+1}<y_{j+1}$. In short, we have $y_{i+1}<y_{j+1}$. After one time impulsive effect, one yields $\varphi\left(y_{i}^{+}\right)=(1+q) y_{i+1}+\sigma<(1+$ q) $y_{j+1}+\sigma=\varphi\left(y_{j}^{+}\right)$. Similarly, for any $y_{i}^{+}, y_{j}^{+} \in\left[y_{\delta E},+\infty\right)$ with 
$y_{i}^{+}<y_{j}^{+}$, the trajectories starting from points $\left((1-\delta) E T, y_{i}^{+}\right)$ and $\left((1-\delta) E T, y_{j}^{+}\right)$first meet $L_{3}$ at points $\left((1-\delta) E T, y_{i}^{\prime}\right)$ and $\left((1-\delta) E T, y_{j}^{\prime}\right)$ with $y_{i}^{\prime}>y_{j}^{\prime}$ and then reach $L_{4}$ at points $\left(E T, y_{i+1}\right)$ and $\left(E T, y_{j+1}\right)$ with $y_{i+1}>y_{j+1}$. After one time impulsive effect, one gets

$$
\begin{aligned}
\varphi\left(y_{i}^{+}\right) & =(1+q) y_{i+1}+\sigma>(1+q) y_{j+1}+\sigma \\
& =\varphi\left(y_{j}^{+}\right) .
\end{aligned}
$$

Thus, the Poincaré map $\varphi$ is increasing on $\left[0, y_{\delta E}\right]$ and decreasing on $\left[y_{\delta E},+\infty\right)$, and the range of $\varphi$ is $[\sigma,(1+q) y((1-$ $\left.\left.\delta) E T, y_{\delta E}\right)+\sigma\right]$.

(II) If $y<y_{\delta E}^{*}$, it follows from the vector fields of system (1) that we have $d y / d t>0$ and $d x / d t>0$, and thus variables $x$ and $y$ both increase along the trajectory. Furthermore, the time for the occurrence of each impulsive effect is denoted by $t_{i}$; then $y(t) \geq y$ holds true for any $t<t_{i}$; that is, $y\left(t_{i}\right) \geq y$. Therefore, $\varphi(y) \geq(1+q) y+\sigma$ provided $y<y_{\delta E}^{*}$.

(III) We need to consider two cases to prove that $\varphi$ is continuous and differentiable, that is, $y<y_{\delta E}$ and $y \geq y_{\delta E}$. For the former, if $y<y_{\delta E}$, then functions $P(x, y) \neq 0$ for any

$$
\begin{gathered}
(x, y) \in\{(1-\delta) E T \leq x \leq E T, 0 \leq y \\
\left.<y\left(t,(1-\delta) E T, y_{\delta E}\right)\right\} \doteq \Upsilon_{(x, y)} .
\end{gathered}
$$

It follows from $\omega(x, y)=Q(x, y) / P(x, y)$ that we obtain that

$$
\begin{aligned}
\frac{\partial \omega}{\partial y}= & \frac{s(1-y / h x)-s y / h x}{r x(1-x / K)-m x y /(A+x)} \\
& +\frac{(1-y / h x)(m x s y /(A+x))}{(r x(1-x / K)-m x y /(A+x))^{2}}
\end{aligned}
$$

is continuous on $\Upsilon_{(x, y)}$. Thus, $\varphi$ is continuously differential when $y<y_{\delta E}$. For the latter, any solution from $\left((1-\delta) E T, y_{0}^{+}\right)$ will turn around the point $P\left((1-\delta) E T, y_{\delta E}\right)$ and cross the line $x=(1-\delta) E T$ when $y<y_{\delta E}$ at $\left((1-\delta) E T, \bar{y}_{0}^{+}\right)$ and then meet $x=E T$. Define the map $\bar{\omega}: \bar{\omega}\left(y_{0}^{+}\right)=$ $\bar{y}_{0}^{+}$. Thereby, $\varphi$ is the composite function of $\varphi$ and $\bar{\omega}$ when $y<y_{\delta E}$. Since $\varphi$ is continuously differential on $\left[0, y_{\delta E}\right), \bar{\omega}$ is continuously differential because of the standard theory of Poincaré application (the Cauchy-Lipschitz Theorem with initial value); it is concluded that $\varphi$ is also continuously differential on $\left[y_{\delta E},+\infty\right)$.

(IV) From (21), we have

$$
\begin{gathered}
\frac{\partial \omega}{\partial y}=\frac{s(1-y / h x)-s y / h x}{r x(1-x / K)-m x y /(A+x)} \\
+\frac{(1-y / h x)(m x s y /(A+x))}{(r x(1-x / K)-m x y /(A+x))^{2}}, \\
\frac{\partial^{2} \omega}{\partial y^{2}}=\frac{2 s y(1-y / h x)(m x /(A+x))^{2}}{(r x(1-x / K)-m x y /(A+x))^{3}}
\end{gathered}
$$

$$
\begin{aligned}
& -\frac{2 s / h x}{r x(1-x / K)-m x y /(A+x)} \\
& +\frac{2 s(1-y / h x)(m x /(A+x))-2 s y m / h(A+x)}{(r x(1-x / K)-m x y /(A+x))^{2}} .
\end{aligned}
$$

It is obvious that $r x(1-x / K)-m x y /(A+x)>0$ and $1-$ $y /(h x)<0$ when $y_{\delta E}^{*} \leq y<y_{\delta E}$, implying that $\partial \omega / \partial y<0$ and $\partial^{2} \omega / \partial y^{2}<0$ hold true for all $y_{\delta E}^{*} \leq y<y_{\delta E}$.

From the theorem of Cauchy and Lipschitz with parameters on the scalar differential equation one gets

$$
\begin{aligned}
\frac{\partial y(x, S)}{\partial S} & =\exp \left[\int_{(1-\delta) E T}^{x} \frac{\partial}{\partial y}\left(\frac{Q(z, y(z, S))}{P(z, y(z, S))}\right) d z\right] \\
& >0,
\end{aligned}
$$

and

$$
\begin{aligned}
& \frac{\partial^{2} y(x, S)}{\partial S^{2}}=\frac{\partial y(x, S)}{\partial S} \\
& \cdot \int_{(1-\delta) E T}^{x} \frac{\partial^{2}}{\partial y^{2}}\left(\frac{Q(z, y(z, S))}{P(z, y(z, S))}\right) \frac{\partial y(x, S)}{\partial S} d z .
\end{aligned}
$$

Based on the above analysis, the inequality $\partial^{2} y(x, S) / \partial S^{2}<$ 0 holds true. Therefore, $\varphi$ is monotonically increasing and concave on $\left[y_{\delta E}^{*}, y_{\delta E}\right)$ (Figure 1).

(V) Since $\varphi(y) \geq(1+q) y+\sigma$ for all $y<y_{\delta E}^{*}$ and $\varphi(y)$ is a decreasing function on $\left[y_{\delta E},+\infty\right)$, then the Poincaré map $\varphi$ has at least one fixed point. If $\varphi\left(y_{\delta E}\right)<y_{\delta E}$, then the Poincaré map $\varphi$ has a fixed point $y_{f}$ with $y_{\delta E}^{*}<y_{f} \leq y_{\delta E}$ (Figure 1(a)). According to the concavity property of $\varphi$, there is not any other fixed point on $\left(y_{\delta E}^{*}, y_{\delta E}\right)$. Since $\varphi$ is decreasing on $\left(y_{\delta E},+\infty\right)$, no other fixed point exists for all $y>y_{\delta E}$. If $\varphi\left(y_{\delta E}\right)>y_{\delta E}$, then it follows from the concavity of $\varphi$ that there is no fixed point when $y \leq y_{\delta E}$, and because $\varphi$ is decreasing on $\left(y_{\delta E},+\infty\right)$, there exists a unique fixed point for $\varphi$ on $\left(y_{\delta E},+\infty\right)$ (Figures $1(\mathrm{c})$ and $\left.1(\mathrm{~d})\right)$.

(VI) Denote the closure of $\Omega$ by

$$
\begin{aligned}
& \bar{\Omega} \\
& =\left\{(x, y) \mid x \geq 0, y \geq 0, y \leq \frac{r}{m}\left(1-\frac{x}{K}\right)(A+x)\right\} .
\end{aligned}
$$

For case $\left(A_{1}\right)$, the set $\bar{\Omega}$ is an invariant set of system (1). In fact, denote

$$
L=y-\frac{r}{m}\left(1-\frac{x}{K}\right)(A+x) .
$$

If the vector field of model (1) is flowing into the boundary $\bar{\Omega}$, then $\bar{\Omega}$ is an invariant set provided

$$
\begin{aligned}
& {\left[(P(x, y), Q(x, y)) \cdot\left(\frac{r}{m}\left(\frac{A+2 x}{K}-1\right), 1\right)\right]_{L=0}} \\
& \quad \leq 0
\end{aligned}
$$


where - denotes the scalar product of two vectors, and the inequality (34) is equivalent to calculate

$$
\begin{aligned}
\left.V(x)\right|_{L=0} \doteq & x\left(r\left(1-\frac{x}{K}\right)-\frac{m y}{A+x}\right) \\
& \cdot \frac{r}{m}\left(\frac{A+2 x}{K}-1\right)+s y\left(1-\frac{y}{h x}\right) \\
= & s y\left(1-\frac{y}{h x}\right),
\end{aligned}
$$

note that $y>y_{\delta E}^{*}$ leads to $\left.V(x)\right|_{L=0}<0$. Besides, $d y / d t<$ 0 if $y>y_{\delta E}^{*}$; it means that for any solution initiating from $\left((1-\delta) E T, y_{0}^{+}\right)$with $y_{0}^{+} \geq y_{\delta E}^{*}$, its vertical coordinate obtains the minimum value at point $\left((1-\delta) E T, y_{\delta E}^{*}\right)$. Thus, $\lim _{y_{k}^{+} \longrightarrow+\infty} g\left(y_{k}^{+}\right)=0$ when $\left((1-\delta) E T, y_{k}^{+}\right) \in \mathscr{P}$. In addition, the solution starting from $((1-\delta) E T, 0)$ reaches the impulsive set in a finite time and then experiences impulsive effect; we have $\lim _{y_{k}^{+} \longrightarrow+\infty} \varphi\left(y_{k}^{+}\right)=\sigma$. It indicates that the horizontal asymptote $y=\sigma$ exists for $\varphi$ when $y_{k}^{+} \longrightarrow+\infty$, as can be seen from Figure 1. This completes the proof.

Since the properties of the Poincare map $\varphi$ have been investigated, the fixed point of $\varphi$ can be discussed which corresponds to the existence of an order-1 limit cycle for system (2). In the following subsection, the boundary order-1 limit cycle will be addressed in detail first.

3.2. Existence and Stability of the Boundary Order-1 Limit Cycle. It follows from system (2) that a boundary order-1 limit cycle exists when $y(t)=0$ if and only if $\sigma=0$. To show this, consider the following subsystem:

$$
\begin{aligned}
& \frac{d x(t)}{d t}=r x(t)\left(1-\frac{x(t)}{K}\right), \quad x(t)<E T, \\
& x\left(t^{+}\right)=(1-\delta) x(t), \quad x(t)=E T .
\end{aligned}
$$

Solving the first equation with initial condition $x\left(0^{+}\right)=(1-$ $\delta) E T$ one yields

$$
x(t)=\frac{(1-\delta) E T K \exp (r t)}{K-(1-\delta) E T+(1-\delta) E T \exp (r t)},
$$

and letting $T$ be the time at which $x(t)$ meets the line $L_{4}$

$$
E T=\frac{(1-\delta) E T K \exp (r t)}{K-(1-\delta) E T+(1-\delta) E T \exp (r t)} .
$$

Solving the above equation with respect to $T$, we have

$$
T=\frac{1}{r} \ln \left(\frac{K-(1-\delta) E T}{(1-\delta)(K-E T)}\right) .
$$

Therefore, there is a boundary order-1 limit cycle for system (2) with period $T$, which is denoted by $\left(x^{T}(t), y^{T}(t)\right)$ and can be described as follows:

$$
\begin{aligned}
& x^{T}(t)=\frac{(1-\delta) E T K \exp (r t)}{K-(1-\delta) E T+(1-\delta) E T \exp (r t)}, \\
& y^{T}(t)=0
\end{aligned}
$$

In the following, we show that the boundary order-1 limit cycle of system (2) is always unstable.

Theorem 6. The boundary order-1 limit cycle $\left(x^{T}(t), 0\right)$ of system (2) is unstable.

Proof. To show this, denote

$$
\begin{aligned}
P(x, y) & =r x\left(1-\frac{x}{K}\right)-\frac{m x y}{A+x}, \\
Q(x, y) & =s y\left(1-\frac{y}{h x}\right), \\
\alpha(x, y) & =-\delta x, \\
\beta(x, y) & =q y+\sigma, \\
\phi(x, y) & =x-E T, \\
\left(x^{T}(T), y^{T}(T)\right) & =(E T, 0), \\
\left(x^{T}\left(T^{+}\right), y^{T}\left(T^{+}\right)\right) & =((1-\delta) E T, 0) .
\end{aligned}
$$

Then

$$
\begin{aligned}
& \frac{\partial P}{\partial x}=r-\frac{2 r x}{K}-\frac{A m y}{(A+x)^{2}}, \\
& \frac{\partial Q}{\partial y}=s-\frac{2 s y}{h x}, \\
& \frac{\partial \alpha}{\partial x}=-\delta, \\
& \frac{\partial \beta}{\partial y}=q, \\
& \frac{\partial \phi}{\partial x}=1, \\
& \frac{\partial \alpha}{\partial y}=\frac{\partial \beta}{\partial x}=\frac{\partial \phi}{\partial y}=0,
\end{aligned}
$$

and

$$
\begin{aligned}
\Delta_{1} & =\frac{P_{+}((\partial \beta / \partial y)(\partial \phi / \partial x)-(\partial \beta / \partial x)(\partial \phi / \partial y)+(\partial \phi / \partial x))+Q_{+}((\partial \alpha / \partial x)(\partial \phi / \partial y)-(\partial \alpha / \partial y)(\partial \phi / \partial x)+\partial \phi / \partial y)}{P(\partial \phi / \partial x)+Q(\partial \phi / \partial y)} \\
& =\frac{P^{+}\left(x^{T}\left(T^{+}\right), y^{T}\left(T^{+}\right)\right)(1+\partial \beta / \partial y)}{P\left(x^{T}(T), y^{T}(T)\right)}=\frac{(1+q)(1-\delta)(K-(1-\delta) E T)}{K-E T} .
\end{aligned}
$$


Moreover, denote $C=(K-(1-\delta) E T) /((1-\delta) E T)$; thus,

$$
\begin{aligned}
& \exp \left(\int_{0}^{T}\left[\frac{\partial P}{\partial x}\left(x^{T}(t), y^{T}(t)\right)+\frac{\partial Q}{\partial y}\left(x^{T}(t), y^{T}(t)\right)\right] d t\right) \\
& \quad=\exp \left(\int_{0}^{T}\left[r+s-\frac{2 r x^{T}(t)}{K}\right] d t\right) \\
& \quad=\exp \left((r+s) T-\left.2 \ln (\exp (r t)+C)\right|_{0} ^{T}\right),
\end{aligned}
$$

and letting $\theta=(K-(1-\delta) E T) /((1-\delta)(K-E T))$ one yields

$$
\begin{aligned}
& \exp \left(\int_{0}^{T}\left[\frac{\partial P}{\partial x}\left(x^{T}(t), y^{T}(t)\right)+\frac{\partial Q}{\partial y}\left(x^{T}(t), y^{T}(t)\right)\right] d t\right) \\
& \quad=\theta^{(r+s) / r}\left(\frac{C+1}{C+\theta}\right)^{2} .
\end{aligned}
$$

The Floquet multiplier $\mu_{2}$ can be calculated as

$$
\begin{aligned}
\mu_{2} & =\Delta_{1} \\
& \cdot \exp \left(\int_{0}^{T}\left[\frac{\partial P}{\partial x}\left(x^{T}(t), y^{T}(t)\right)+\frac{\partial Q}{\partial y}\left(x^{T}(t), y^{T}(t)\right)\right] d t\right) \\
& =(1+q)\left(\frac{K-(1-\delta) E T}{(1-\delta)(K-E T)}\right)^{s / r} .
\end{aligned}
$$

It is obvious that

$$
\frac{K-(1-\delta) E T}{(1-\delta)(K-E T)}>1
$$

holds true. Therefore, it follows from $\left|\mu_{2}\right|>1$ that the boundary order-1 limit cycle $\left(x^{T}(t), 0\right)$ of system (2) is unstable. This completes the proof.

If we fix the parameter values as shown in Figure 2, then it can be seen that the boundary order-1 limit cycle of system (2) is unstable and an interior order-1 limit cycle is generated. In addition, all solutions tend to the interior order-1 limit cycle, as shown in Figure 2.

3.3. Existence and Stability of Limit Cycles for $\sigma>0$. In this subsection, the existence of the order- $k$ limit cycle of system (2) under case $\left(A_{1}\right)$ will be investigated, which is equivalent to the discussion of fixed point of the Poincare map. For simplicity, the generalized result for the stability of the order1 limit cycle $(\xi(t), \eta(t))$ will be provided first. To this end, we have the following generalized result.

Theorem 7. The order-1 limit cycle $(\xi(t), \eta(t))$ is orbitally asymptotically stable when and only when

$$
\left|\frac{(1+q)(1-\delta)\left(r(1-(1-\delta) E T / K)-m\left((1+q) \eta_{0}+\sigma\right) /(A+(1-\delta) E T)\right)}{r(1-E T / K)-m \eta_{0} /(A+E T)} \exp \left(\int_{0}^{T} G(t) d t\right)\right|<1,
$$

where $G(t)=r+s-2 r \xi(t) / K-m \eta(t) /(A+\xi(t))^{2}-2 s \eta(t) / h \xi(t)$.

Proof. Without loss of generality, it is assumed that the order-1 limit cycle with period $T$ passes through the points
$M\left(E T, \eta_{0}\right)$ and $M^{+}\left((1-\delta) E T,(1+q) \eta_{0}+\sigma\right)$. Based on the proofs of Theorem 6 , the Floquet multiplier $\mu_{2}$ can be calculated as

$$
\begin{aligned}
\mu_{2} & =\Delta_{1} \exp \left(\int_{0}^{T}\left[\frac{\partial P}{\partial x}(\xi(t), \eta(t))+\frac{\partial Q}{\partial y}(\xi(t), \eta(t))\right] d t\right) \\
& =\frac{(1+q)(1-\delta)\left(r(1-(1-\delta) E T / K)-m\left((1+q) \eta_{0}+\sigma\right) /(A+(1-\delta) E T)\right)}{r(1-E T / K)-m \eta_{0} /(A+E T)} \exp \left(\int_{0}^{T} G(t) d t\right)
\end{aligned}
$$

Therefore, it follows from the inequality (48) that $\left|\mu_{2}\right|<$ 1 ; that is, the order-1 limit cycle $(\xi(t), \eta(t))$ is orbitally asymptotically stable.

For case $\left(A_{1}\right)$, as mentioned before, any solution initiating from $\left((1-\delta) E T, y_{0}^{+}\right)$with $y_{0}^{+} \in D_{0}$ experiences infinitely many pulses; we write the corresponding impulsive point series as $y_{n}^{+}(n=1,2, \ldots)$ with $y_{n}^{+}=\varphi^{n}\left(y_{0}^{+}\right)$. In the following, what we want to show is the existence of the fixed point of the Poincaré $\operatorname{map} \varphi$, which corresponds to the existence of the order- $k(k \geq$ 1) limit cycle for system (2).

Theorem 8. If $\varphi\left(y_{\delta E}\right)<y_{\delta E}$, then the Poincaré map $\varphi$ has a unique fixed point $y_{f}$ with $y_{\delta E}^{*}<y_{f} \leq y_{\delta E}$ which is globally asymptotically stable, and it implies system (2) exists with an order-1 limit cycle.

Proof. From the property (V) of the Poincaré map, as shown in Theorem 5, if $\varphi\left(y_{\delta E}\right)<y_{\delta E}$, then there is a unique fixed point $y_{f}$ with $y_{\delta E}^{*}<y_{f} \leq y_{\delta E}$ for the Poincaré map. Further, the results of Theorem 7 indicate that this unique order-1 limit cycle is orbitally asymptotically stable. To show the global stability of the order-1 limit cycle, we only need to show that this unique order-1 limit cycle is globally attractive.

For any solution starting from $\left((1-\delta) E T, y_{0}^{+}\right)$, if $y_{0}^{+} \epsilon$ $\left[0, y_{f}\right)$, according to the properties (II) and (IV) of the Poincaré map, then $y_{f}>\varphi\left(y_{0}^{+}\right)>y_{0}^{+}$holds true, 


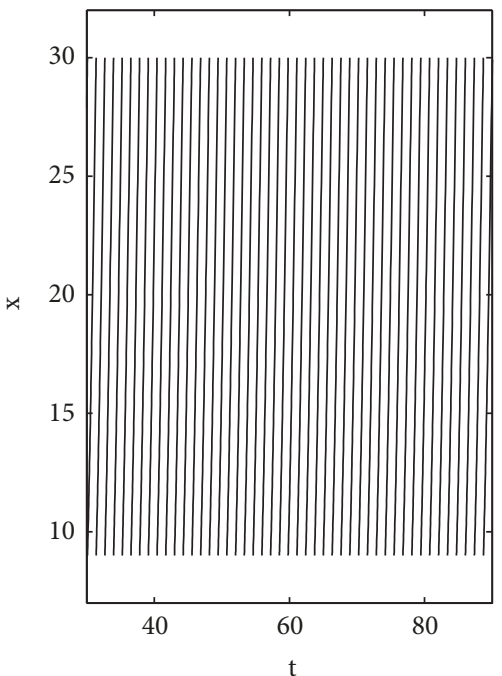

(a)

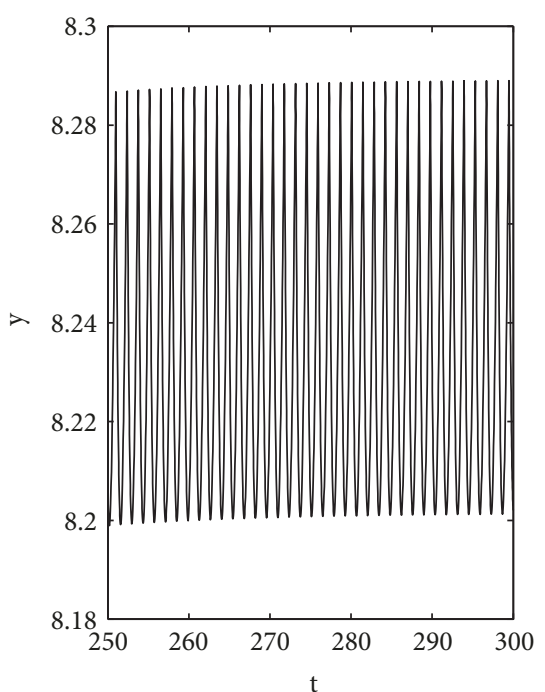

(b)

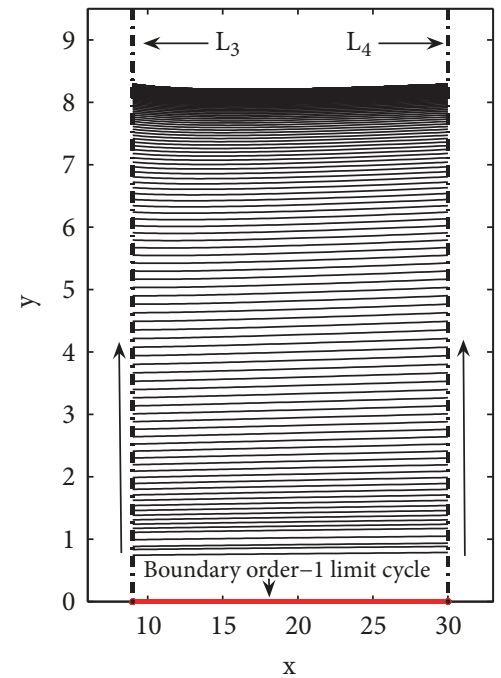

(c)

Figure 2: $(\mathrm{a}-\mathrm{c})$ The boundary order-1 limit cycle $\left(x^{T}(t), 0\right)$ is unstable and an order-1 limit cycle is generated. The red line represents the boundary order-1 limit cycle. The parameters are fixed as $r=1.5, K=50, m=0.15, A=0.5, s=0.05, h=0.5, E T=30, \delta=0.7, \delta=0$, and $\sigma=0$.

which implies that $\varphi^{n}\left(y_{0}^{+}\right)$is monotonically increasing as $n$ increases. Therefore, $\lim _{n \rightarrow+\infty} \varphi^{n}\left(y_{0}^{+}\right)=y_{f}$, as shown in Figure 1(a).

For any solution starting from $\left((1-\delta) E T, y_{0}^{+}\right)$, if $y_{0}^{+}>y_{f}$, then there are two possible cases for $\varphi^{n}\left(y_{0}^{+}\right)$: (a) $\varphi^{n}\left(y_{0}^{+}\right)>y_{f}$ for all $n$; (b) $\varphi^{n}\left(y_{0}^{+}\right)>y_{f}$ does not hold true for all $n$. For the former, it follows from $\varphi\left(y_{0}^{+}\right)<y_{0}^{+}$that $\varphi^{n}\left(y_{0}^{+}\right)$is monotonically decreasing as $n$ increases, and $\lim _{n \longrightarrow+\infty} \varphi^{n}\left(y_{0}^{+}\right)=y_{f}$. For the latter, assume that there exists a smallest positive integer $n_{1}$ such that $\varphi^{n_{1}}\left(y_{0}^{+}\right)<y_{f}$. From the analysis of case (a), it suggests that $\varphi^{n_{2}}\left(y_{0}^{+}\right)$is monotonically increasing as $n_{2}$ increases $\left(n_{2}\right.$ is a positive integer and $\left.n_{2}>n_{1}\right)$, and $\lim _{n_{2} \rightarrow+\infty} \varphi^{n_{2}}\left(y_{0}^{+}\right)=y_{f}$. Therefore, the unique order-1 limit cycle is globally attracting and consequently is globally asymptotically stable. This completes the proof.

Remark 9. In particular, if $\varphi\left(y_{\delta E}\right)=y_{\delta E}$, then it follows from Theorem 8 that there is a unique globally asymptotically stable fixed point $y_{\delta E}$ for the Poincaré map, which refers to a unique globally asymptotically stable order-1 limit cycle for system (2).

Theorem 10. If $\varphi\left(y_{\delta E}\right)>y_{\delta E}$ and $\varphi^{2}\left(y_{\delta E}\right) \geq y_{\delta E}$, then there is a stable fixed point or a period two-point cycle for the Poincaré map, and it indicates that system (2) does not permit an order$k(k \geq 3)$ limit cycle except for the stable order-1 limit cycle or order-2 limit cycle.

Proof. For any $y_{0}^{+} \in\left[0, y_{\delta E}\right]$, it follows from the properties of the Poincare map that there does not exist a fixed point for $\varphi$ on $\left[0, y_{\delta E}\right]$, and $\varphi$ is monotonically increasing on $\left[0, y_{\delta E}\right]$. Thus, there will be an integer $i$ such that $y_{i-1}^{+}<y_{\delta E}$ and $y_{i}^{+} \geq y_{\delta E}$. Since $\varphi$ is monotonically increasing on $\left[0, y_{\delta E}\right]$, then it is obvious that $y_{i}^{+}=\varphi\left(y_{i-1}^{+}\right) \leq \varphi\left(y_{\delta E}\right)$. Therefore, we have $y_{i}^{+} \in\left[y_{\delta E}, \varphi\left(y_{\delta E}\right)\right]$. In addition, for any $y_{0}^{+}>y_{\delta E}$, after one time impulsive effect, $y_{1}^{+}=\varphi\left(y_{0}^{+}\right) \leq \varphi\left(y_{\delta E}\right)$ because $\varphi$ is monotonically decreasing on $\left[y_{\delta E},+\infty\right)$. Therefore, there will be an integer $i \geq 1$ such that $y_{i}^{+} \in\left[y_{\delta E}, \varphi\left(y_{\delta E}\right)\right]$.

Since $\varphi$ is monotonically decreasing on $\left[y_{\delta E},+\infty\right)$ and $\varphi^{2}$ is monotonically increasing on $\left[y_{\delta E},+\infty\right)$, one yields

$$
\begin{aligned}
\varphi\left(\left[y_{\delta E}, \varphi\left(y_{\delta E}\right)\right]\right) & =\left[\varphi^{2}\left(y_{\delta E}\right), \varphi\left(y_{\delta E}\right)\right] \\
& \subset\left[y_{\delta E}, \varphi\left(y_{\delta E}\right)\right] .
\end{aligned}
$$

Without loss of generality, for any $y_{0}^{+} \in\left[y_{\delta E}, \varphi\left(y_{\delta E}\right)\right]$, assume that $y_{1}^{+}=\varphi\left(y_{0}^{+}\right) \neq y_{0}^{+}, y_{2}^{+}=\varphi^{2}\left(y_{0}^{+}\right) \neq y_{0}^{+}$, and $y_{n}^{+}=\varphi^{n}\left(y_{0}^{+}\right)$. For the relations among $y_{\delta E}, \varphi\left(y_{\delta E}\right), y_{0}^{+}, y_{1}^{+}$, and $y_{2}^{+}$, there may be the following four possible cases:

$\left(C_{1}\right) y_{\delta E} \leq y_{2}^{+}<y_{0}^{+}<y_{1}^{+} \leq \varphi\left(y_{\delta E}\right)$. In this case, it can be seen that $y_{1}^{+}=\varphi\left(y_{0}^{+}\right)<\varphi\left(y_{2}^{+}\right)=y_{3}^{+}$and $y_{4}^{+}=\varphi\left(y_{3}^{+}\right)<$ $\varphi\left(y_{1}^{+}\right)=y_{2}^{+}$; thus we have $y_{4}^{+}<y_{2}^{+}<y_{0}^{+}<y_{1}^{+}<y_{3}^{+}$. By induction one obtains

$$
\begin{aligned}
y_{\delta E} & \leq \cdots<y_{2 n+2}^{+}<y_{2 n}^{+}<\cdots<y_{2}^{+}<y_{0}^{+}<y_{1}^{+}<\cdots \\
& <y_{2 n-1}^{+}<y_{2 n+1}^{+}<\cdots \leq \varphi\left(y_{\delta E}\right) .
\end{aligned}
$$

$\left(C_{2}\right) y_{\delta E} \leq y_{0}^{+}<y_{2}^{+}<y_{1}^{+} \leq \varphi\left(y_{\delta E}\right)$. Similarly, $\varphi\left(y_{1}^{+}\right)=$ $y_{2}^{+}<y_{3}^{+}=\varphi\left(y_{2}^{+}\right)<\varphi\left(y_{0}^{+}\right)=y_{1}^{+}$and $y_{2}^{+}=\varphi\left(y_{1}^{+}\right)<\varphi\left(y_{3}^{+}\right)=$ $y_{4}^{+}<y_{3}^{+}=\varphi\left(y_{2}^{+}\right)=y_{1}^{+}$, so $y_{0}^{+}<y_{2}^{+}<y_{4}^{+}<y_{3}^{+}<y_{1}^{+}$. By induction one obtains

$$
\begin{aligned}
y_{\delta E} & \leq y_{0}^{+}<y_{2}^{+}<\cdots<y_{2 n}^{+}<y_{2 n+2}^{+}<\cdots<y_{2 n+1}^{+} \\
& <y_{2 n-1}^{+}<\cdots<y_{1}^{+} \leq \varphi\left(y_{\delta E}\right) .
\end{aligned}
$$


$\left(C_{3}\right) y_{\delta E} \leq y_{1}^{+}<y_{2}^{+}<y_{0}^{+} \leq \varphi\left(y_{\delta E}\right)$. By using the same method as case $\left(C_{2}\right)$ one obtains

$$
\begin{aligned}
y_{\delta E} & \leq y_{1}^{+}<\cdots<y_{2 n-1}^{+}<y_{2 n+1}^{+}<\cdots<y_{2 n+2}^{+}<y_{2 n}^{+} \\
& <\cdots<y_{2}^{+}<y_{0}^{+} \leq \varphi\left(y_{\delta E}\right) .
\end{aligned}
$$

$\left(C_{4}\right) y_{\delta E} \leq y_{1}^{+}<y_{0}^{+}<y_{2}^{+} \leq \varphi\left(y_{\delta E}\right)$. By using the same method as case $\left(C_{1}\right)$ one obtains

$$
\begin{aligned}
y_{\delta E} & \leq \cdots<y_{2 n+1}^{+}<y_{2 n-1}^{+}<\cdots<y_{1}^{+}<y_{0}^{+}<y_{2}^{+}<\cdots \\
& <y_{2 n}^{+}<y_{2 n+2}^{+}<\cdots \leq \varphi\left(y_{\delta E}\right) .
\end{aligned}
$$

From the above analysis, for cases $\left(C_{2}\right)$ and $\left(C_{3}\right)$, there either exists a unique $y_{f}$ such that $\lim _{n \rightarrow+\infty} y_{2 n+1}^{+}=$ $\lim _{n \longrightarrow+\infty} y_{2 n}^{+}=y_{f}$ with $y_{f} \in\left[y_{\delta E}, \varphi\left(y_{\delta E}\right)\right]$, or there exist $y_{f}^{1}$ and $y_{f}^{2}$ such that $\lim _{n \longrightarrow+\infty} y_{2 n+1}^{+}=y_{f}^{1}$ and $\lim _{n \longrightarrow+\infty} y_{2 n}^{+}=y_{f}^{2}$ with $y_{f}^{1}, y_{f}^{2} \in\left[y_{\delta E}, \varphi\left(y_{\delta E}\right)\right]$ and $y_{f}^{1} \neq y_{f}^{2}$. Therefore, system (2) does not have an order- $k(k \geq 3)$ limit cycle except for the order-1 limit cycle or order-2 limit cycle (Figures $1(\mathrm{c})$ and $1(\mathrm{~d}))$. However, for cases $\left(C_{1}\right)$ and $\left(C_{4}\right)$, system (2) only has an order-2 limit cycle. This completes the proof.

From Theorem 10, the sufficient conditions for the existence and stability of an order-1 or order-2 limit cycle of system (2) are provided when $\varphi\left(y_{\delta E}\right)>y_{\delta E}$. In the following, what we want to do is to show the global stability of the order1 limit cycle for system (2).

Theorem 11. If $\varphi\left(y_{\delta E}\right)>y_{\delta E}$, then the order-1 limit cycle of system (2) is globally stable if and only if $\varphi^{2}(y)>y$ for all $y \in\left[y_{\delta E}, y_{f}\right)$.

Proof. From the proof of Theorem 5, if $\varphi\left(y_{\delta E}\right)>y_{\delta E}$, then there is a unique fixed point $y_{f}$ for $\varphi$, and further $\varphi(y)<y$ for all $y \in\left(y_{f},+\infty\right), \varphi(y)>y$ for all $y \in\left[0, y_{f}\right)$. According to the concavity of $\varphi$ we know $\varphi(y)$ reaches its maximal value at $y_{\delta E}$ with $y_{\delta E} \in\left(0, y_{f}\right)$.

Now we prove that if $\varphi^{2}(y)>y$ for all $y \in\left[y_{\delta E}, y_{f}\right)$, then the order-1 limit cycle of system (2) is globally stable. To this end, the following three cases will be considered: $\left(c_{1}\right) y \in$ $\left[y_{\delta E}, y_{f}\right),\left(c_{2}\right) y \in\left[0, y_{\delta E}\right)$, and $\left(c_{3}\right) y \in\left(y_{f},+\infty\right)$.

For case $\left(c_{1}\right)$, because $\varphi(y)$ is monotonically decreasing when $y \in\left[y_{\delta E}, y_{f}\right), \varphi\left(y_{\delta E}\right) \geq \varphi(y)>y_{f}$. It is easy to see that $\varphi^{2}(y)$ is monotonically increasing on $\left[y_{\delta E}, y_{f}\right)$; thus $y<$ $\varphi^{2}(y)<y_{f}$ holds true. By induction one obtains $\varphi^{2(k-1)}(y)<$ $\varphi^{2 k}(y)<y_{f}(k \geq 1$ and is a positive integer). Therefore, $\varphi^{2 k}(y)$ is monotonically increasing with $\lim _{k \rightarrow+\infty} \varphi^{2 k}(y)=$ $y_{f}$, and the results for $\varphi^{2 k-1}(y)$ can be proved by using the same methods.

For case $\left(c_{2}\right)$, it follows from the proof of Theorem 10 that there is a positive integer $i$ such that $\varphi^{i}(y) \in\left[y_{\delta E}, y_{f}\right)$ or $\varphi^{i}(y) \in\left(y_{f},+\infty\right)$. For the former, by using the same methods as case $\left(c_{1}\right)$, it can be proved that $\varphi^{i+2 k}(y)$ is monotonically increasing with $\lim _{k \longrightarrow+\infty} \varphi^{i+2 k}(y)=y_{f}$. For the latter, it is obvious that $\varphi^{i+1}(y) \in\left(y_{\delta E}, y_{f}\right)$ must hold true. Therefore, we get $\lim _{k \rightarrow+\infty} \varphi^{(i+1)+2 k}(y)=y_{f}$.
For case $\left(c_{3}\right)$, assume that the solution starting from $((1-$ $\delta) E T, y)$ experiences $k$ impulsive effects; then for $\varphi^{k}(y)$ there may be two cases; that is, $\varphi^{k}(y)>y_{f}$ holds true for all $k$ or there will be a positive integer $i$ such that $\varphi^{i}(y) \in\left(0, y_{\delta E}\right)$ $\left(\operatorname{or} \varphi^{i}(y) \in\left[y_{\delta E}, y_{f}\right)\right)$. For the former, $\varphi^{k}(y)$ is monotonically decreasing due to $\varphi(y)<y$ with $\lim _{k \longrightarrow+\infty} \varphi^{k}(y)=y_{f}$. For the latter, the results are also true by using the same methods as cases $\left(c_{1}\right)$ and $\left(c_{2}\right)$.

Finally, we prove that if the order-1 limit cycle of system (2) is globally stable, then $\varphi^{2}(y)>y$ holds true for all $y \in$ $\left[y_{\delta E}, y_{f}\right)$. Otherwise, if there exists at least one $y^{+} \in\left[y_{\delta E}, y_{f}\right)$ such that $\varphi^{2}\left(y^{+}\right)<y^{+}$, then in a small region of $y_{f}$ there exists a $y^{++}$such that $\varphi^{2}\left(y^{++}\right)>y^{++}$. According to the continuity of $\varphi^{2}$, there exists another fixed point $y_{f}^{*} \in\left(y^{+}, y^{++}\right)$for $\varphi^{2}$. This is a contradiction with the global stability of the order-1 limit cycle. This completes the proof.

Theorem 12. Let $y_{m}^{+}=\min \left\{y^{+}: \varphi\left(y^{+}\right)=y_{\delta E}\right\}$. If $\varphi\left(y_{\delta E}\right)>$ $y_{\delta E}$ and $\varphi^{2}\left(y_{\delta E}\right)<y_{m}^{+}$, then system (2) exists with an order-3 limit cycle.

Proof. If $\varphi\left(y_{\delta E}\right)>y_{\delta E}$, it has been proved that there exists a unique fixed point $y_{f}$ for $\varphi$ on interval $\left(y_{\delta E}, \varphi\left(y_{\delta E}\right)\right)$. In the following, we only need to find a $y \in[0,+\infty)$ so that $\varphi^{3}(y)=y$ and $\varphi(y) \neq y$. It is clear that $\varphi^{3}(y)$ is continuous on $[0,+\infty)$ because $\varphi(y)$ is continuous on $[0,+\infty)$. Based on these assumptions, the Poincaré map $\varphi^{3}$ satisfies

$$
\varphi^{3}\left(y_{m}^{+}\right)=\varphi^{2}\left(y_{\delta E}\right)<y_{m}^{+} \text {, and } \varphi^{3}(0)=\varphi^{2}(\sigma)>0,
$$

thus, there exists a fixed point $y_{f}^{*}$ such that $\varphi^{3}\left(y_{f}^{*}\right)=y_{f}^{*}$ with $y_{f}^{*} \in\left(0, y_{m}^{+}\right)$. Moreover, $y_{m}^{+}<y_{\delta E}$ and $y_{f}>y_{\delta E}$ hold true. Therefore, $\varphi^{3}$ has a fixed point which corresponds to an order-3 limit cycle for system (2) (Figure 3). This completes the proof.

Remark 13. Note that the conditions for the existence of an order-3 limit cycle have been provided in Theorem 12, and it suggests that system (2) exists with order- $k(k \geq 3)$ limit cycles, which implies chaos [31,32].

Based on case $\left(A_{1}\right)$, we have not only studied the existence and stability of the order-1 limit cycle, but also provided the conditions for the existence of the order$k(k \geq 2)$ limit cycle. In the following, numerical simulations will be carried out to verify the theoretical results. Fixed parameters are shown in Figure 3; it is shown that system (2) exists with an order-1 limit cycle (Figure 3(a)), an order-2 limit cycle (Figure 3(b)), an order-3 limit cycle (Figure 3(c)), and chaos (Figure 3(d)) when $\sigma$ decreases, which confirms our main results. Note that Theorem 7 provides a condition for the orbitally asymptotically stable of an order-1 limit cycle; an example is given to show the possibility that parameters satisfy Theorem 7 . In the case of an order-1 limit cycle (Figure $3(\mathrm{a})), \xi(t) \leq K$ holds and 


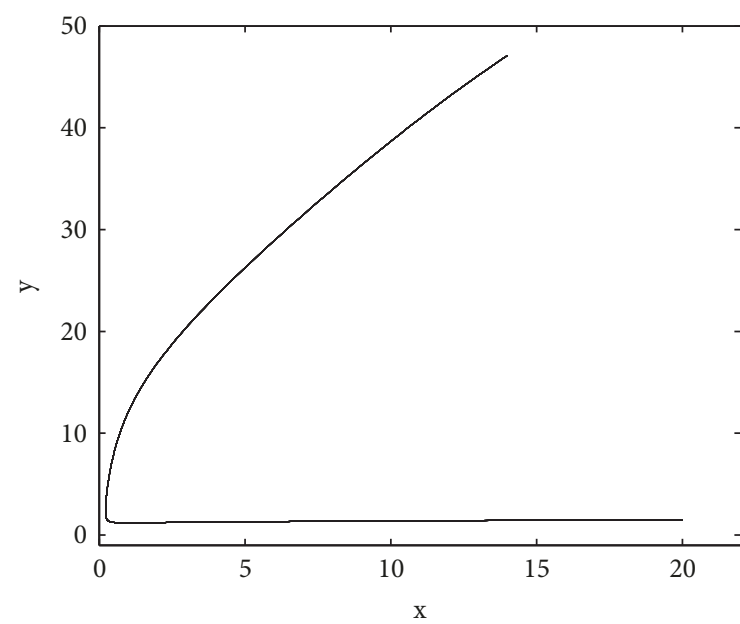

(a)

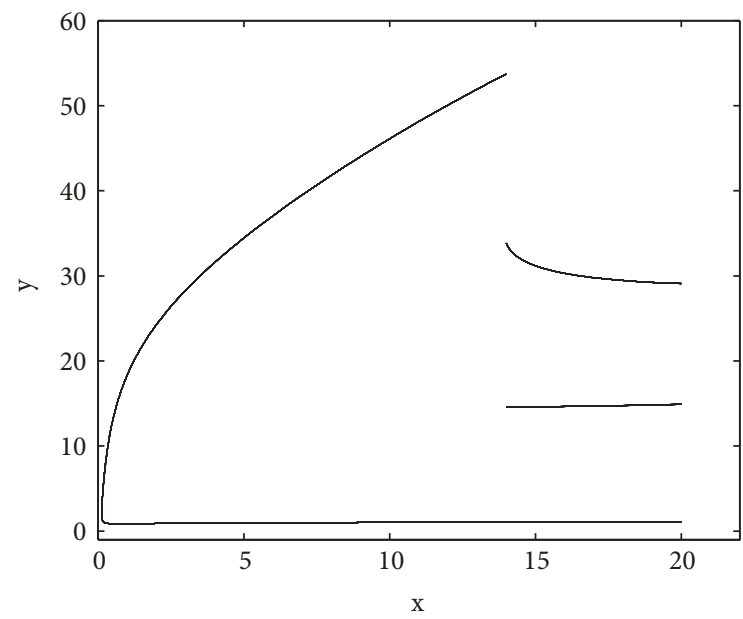

(c)

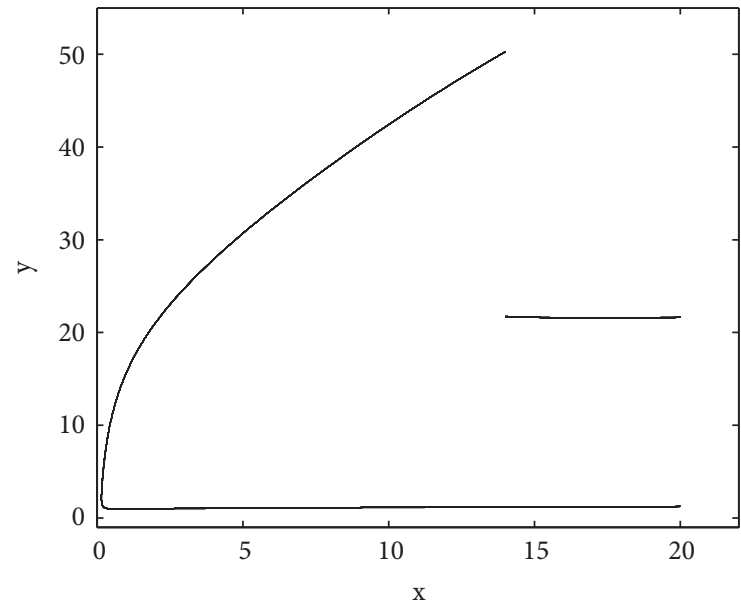

(b)

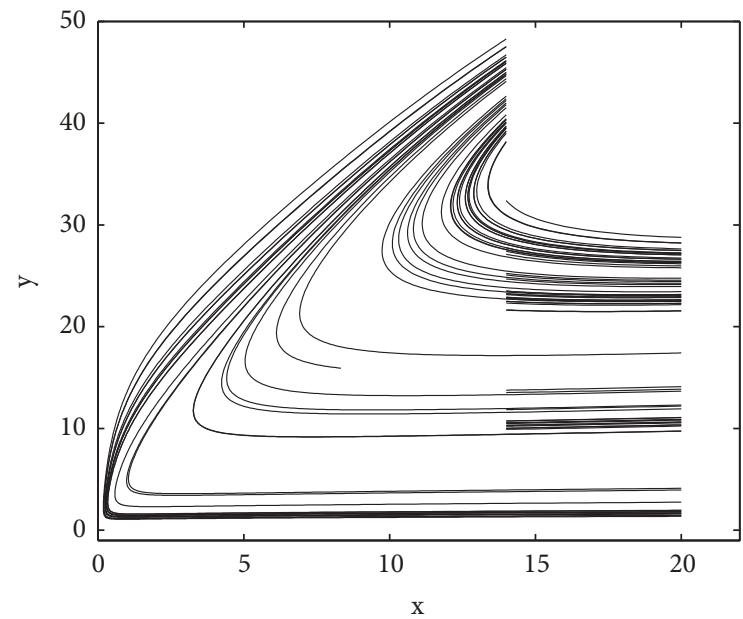

(d)

FIGURE 3: Order-k limit cycles of system (2). (a) Order-1 limit cycle with $\sigma=45$; (b) order-2 limit cycle with $\sigma=20$; (c) order-3 limit cycle with $\sigma=13$; (d) chaos with $\sigma=8$. All other parameter values were fixed as $r=0.5, K=50, m=0.15, A=0.5, s=0.043, h=1.25, E T=20$, $\delta=0.3$, and $q=0.4$.

$G(t)=r+s-2 r \xi(t) / K-m \eta(t) /(A+\xi(t))^{2}-2 s \eta(t) / h \xi(t) \leq$

$r+s-2 r \xi(t) / K \leq s-r ;$ thus

$$
\begin{aligned}
\left|\mu_{2}\right| & =\left|\frac{(1+q)(1-\delta)\left(r(1-(1-\delta) E T / K)-m\left((1+q) \eta_{0}+\sigma\right) /(A+(1-\delta) E T)\right)}{r(1-E T / K)-m \eta_{0} /(A+E T)} \exp \left(\int_{0}^{T} G(t) d t\right)\right| \\
& \leq\left|\frac{(1+q)(1-\delta)\left(r(1-(1-\delta) E T / K)-m\left((1+q) \eta_{0}+\sigma\right) /(A+(1-\delta) E T)\right)}{r(1-E T / K)-m \eta_{0} /(A+E T)}\right|\left|\exp \left(\int_{0}^{T} s-r d t\right)\right| .
\end{aligned}
$$

then we substitute the parameter values of Figure 3(a) into the above equation:

$$
\left|\mu_{2}\right| \leq|-0.43|\left|\exp \left(\int_{0}^{27.1}-0.457 d t\right)\right| \approx \frac{0.43}{228662}
$$$$
\ll 1 \text {. }
$$

It follows from Theorem 7 that the order-1 limit cycle $(\xi(t), \eta(t))$ is orbitally asymptotically stable (Figure $3(\mathrm{a})$ ).

Now we choose control parameters as bifurcation parameters to show the complex dynamics of system (2), In Figure 4, the simulation reveals complexities of system (2). In Figure 4(a), as $\sigma$ increases, period-doubling bifurcations 


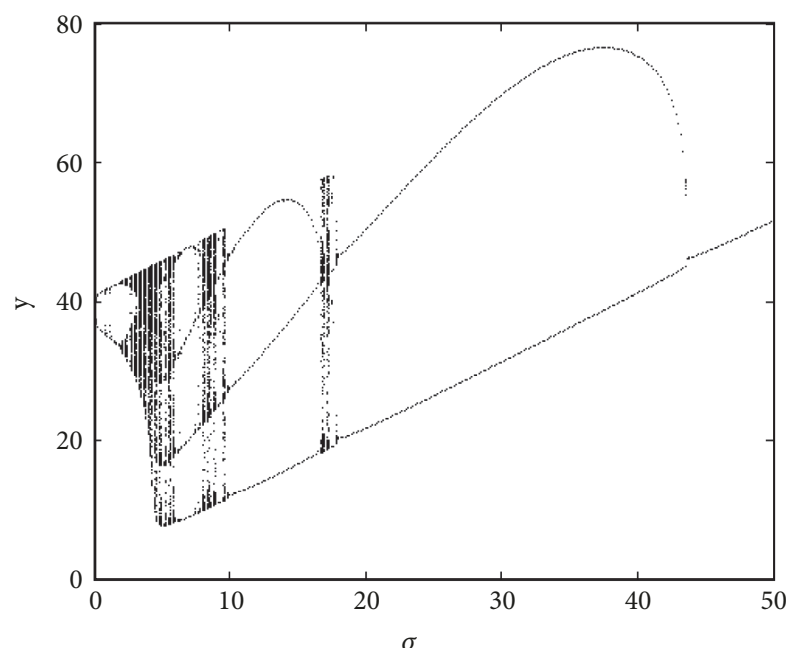

(a)

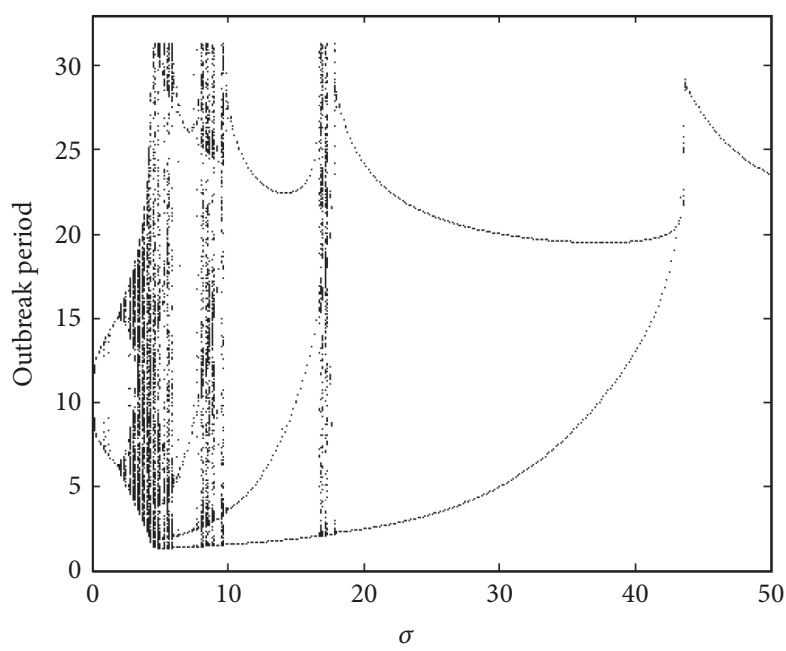

(c)

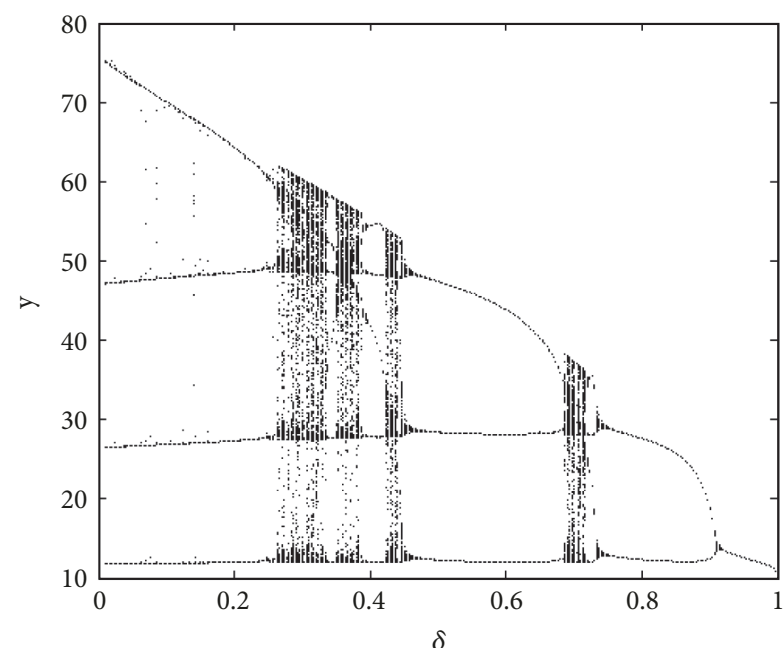

(b)

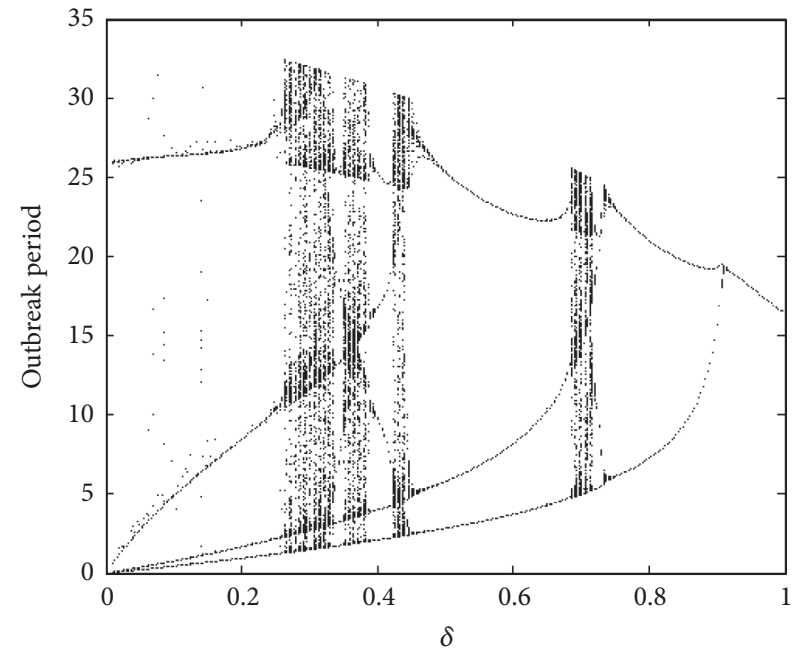

(d)

FIGURE 4: (a) and (c) bifurcations of system (2) for case $\left(A_{1}\right)$ with respect to control parameter $\sigma$, the parameter values were fixed as $r=0.5$, $K=50, m=0.15, A=0.5, s=0.043, h=1.25, E T=20, \delta=0.3$, and $q=0.4$; (b) and (d) bifurcations of system (2) for case $\left(A_{1}\right)$ with respect to $\delta$ when $r=0.5, K=60, m=0.15, A=0.5, s=0.05, h=1.333, E T=25, \sigma=10$, and $q=0.4$.

lead system (2) to chaos. When $\sigma$ continues to increase, period-halving bifurcations lead system (2) to an order-4 limit cycle. Furthermore, system (2) exhibits sharp changes from order- $(n+1)$ limit cycles to order- $n(n=1,2,3)$ limit cycles via period-decreasing bifurcations with chaotic bands. Moreover, as $\delta$ increases system (2) also exhibits perioddecreasing bifurcations with sharp changes (Figure 4(b)). In addition, the bifurcation diagrams of the outbreak period of the pest population with respect to $\sigma$ and $\delta$ are also indicated in Figures 4(c) and 4(d). All these confirm that the theorems of case $\left(A_{1}\right)$ are correct and further indicate that small changes in $\sigma$ and $\delta$ would result in the pests and enemies oscillate periodically.

\section{Complexity of Poincaré Map for Case $\left(A_{2}\right)$}

4.1. Complex Domains of Poincaré Map. For case $\left(A_{2}\right), L_{4}$ is located to the right of the equilibrium $E^{*}$, while the locations of $L_{3}$ could vary. According to Lemma 4, the equilibrium $E^{*}$ of system (2) could be stable or unstable under certain conditions. Without loss of generality, unless otherwise specified, we assume that $E^{*}$ is a focus for system (2) in the following, and let Is and Ps denote the impulsive set and phase set.

For case (i) of Lemma $4, E^{*}$ is a globally stable focus. To derive domains for Is and Ps, we only need to consider the case $E T \geq x^{*}$. For this case, as shown in Figure 5(a), there is a solution denoted by $\Lambda_{2}$ which is tangential to the line $L_{4}$ at the point $Q$, and then $\Lambda_{2}$ intersects the line $L_{2}$ at a point $P_{1}\left(x_{P_{1}}, y_{P_{1}}\right)$ such that $\Lambda_{2}$ is tangential to $L_{2}$ at this point. If $(1-$ $\delta) E T<x_{P_{1}}$, then the curve $\Lambda_{1}$ starting from the point $P((1-$ $\left.\delta) E T, y_{\delta E}\right)$ which is tangential to $L_{2}$ will meet $L_{4}$ at a point $Q_{1}\left(E T, y_{Q_{1}}\right)$. So we derive Is $\mathscr{I}_{1}=\left\{(x, y) \in R^{2} \mid x=E T, 0 \leq\right.$ $\left.y \leq y_{Q_{1}},\right\}$ and Ps $\mathscr{P}_{1}=\left\{\left(x^{+}, y^{+}\right) \in R^{2} \mid x^{+}=(1-\delta) E T, y^{+} \in\right.$ $\left.D_{1}\right\}$ with $D_{1}=\left[\sigma,(1+q) y_{Q_{1}}+\sigma\right]$. If $(1-\delta) E T \geq x_{P_{1}}$, then 


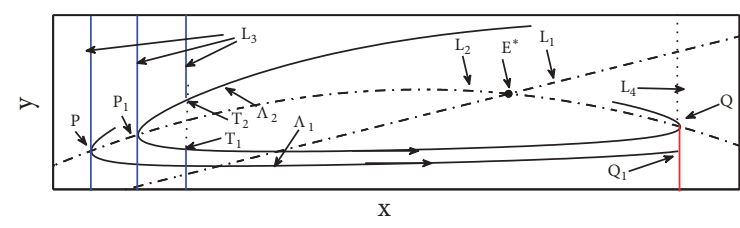

(a)

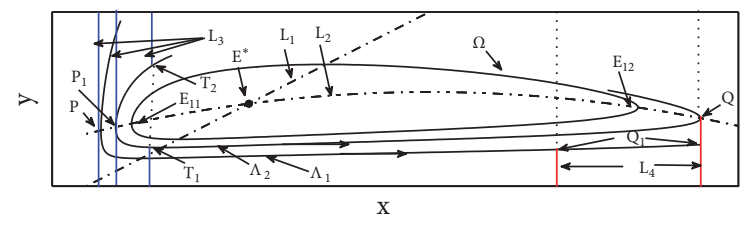

(b)

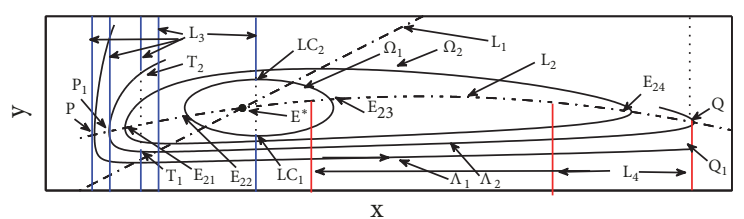

(c)

Figure 5: The definition domains of phase set and impulsive set. (a) Case (i) of Lemma 4, that is, there exists a globally stable focus of system (2). (b) Case (ii) of Lemma 4, that is, there exists a unique limit cycle $\Omega$ of system (2). (c) Case (iii) of Lemma 4 , that is, there exists two limit cycles $\Omega_{1}$ and $\Omega_{2}$ of system (2). The blue lines represent the phase sets and the red line denotes the impulsive sets under different cases.

$L_{3}$ must intersect $\Lambda_{2}$ at two points $T_{1}\left((1-\delta) E T, y_{\min }^{P}\right)$ and $T_{2}\left((1-\delta) E T, y_{\text {max }}^{P}\right)$. Thus, Is is $\mathscr{I}$ and we obtain the Ps $\mathscr{P}_{2}=$ $\left\{\left(x^{+}, y^{+}\right) \in R^{2} \mid x^{+}=(1-\delta) E T, y^{+} \in D_{2}\right\}$, where $D_{2}=$ $\left\{\left[y_{\text {max }}^{P},+\infty\right) \cup\left[0, y_{\text {min }}^{P}\right]\right\}$.

For case (ii) of Lemma $4, E^{*}$ is an unstable focus and the unique stable limit cycle is denoted by $\Omega$, which intersects $L_{2}$ at two points $E_{11}\left(x_{11}, y_{11}\right)$ and $E_{12}\left(x_{12}, y_{12}\right)$ with $x_{11}<$ $x_{12}$; see Figure 5(b). In order to provide the domains for the Poincaré map, based on the positions of $(1-\delta) E T, E T, x_{11}$, and $x_{12}$, we need to consider three possible cases:

$$
\begin{aligned}
&\left(B_{1}\right) \quad(1-\delta) E T \leq x_{11}, \\
& E T<x_{12}, \\
&\left(B_{2}\right) \quad E T \geq x_{12}, \\
&\left(B_{3}\right) \quad x_{11}<(1-\delta) E T<E T<x_{12} .
\end{aligned}
$$

For case $\left(B_{1}\right)$, from the analysis for case (i) of Lemma 4 , we obtain Is $\mathscr{I}_{1}$ and Ps $\mathscr{P}_{1}$. Similarly, for case $\left(B_{2}\right)$, if $(1-$ $\delta) E T<x_{P_{1}}$, then we obtain Is $\mathscr{I}_{1}$ and Ps $\mathscr{P}_{1}$ (Figure 5(b)). If $(1-\delta) E T \geq x_{P_{1}}$, then Is is $\mathscr{I}$ and Ps is $\mathscr{P}_{2}$. For case $\left(B_{3}\right)$, note that $L_{3}$ and $L_{4}$ are located between $x_{11}$ and $x_{12}$, any trajectory starting from $\mathscr{P}$ (not include $E^{*}$ ) may meet impulsive set or have multiple intersection points with $L_{3}$ before meeting impulsive set, thereafter undergoes impulsive effects. Thus, we obtain Is $\mathscr{I}$ and Ps $\mathscr{P} /\left\{E^{*}\right\}$, and $\mathscr{P} /\left\{E^{*}\right\}$ indicates $E^{*}$ is excluded from $\mathscr{P}$.

For case (iii) of Lemma 4 (for details see Figure 5(c)), it is revealed that $E^{*}$ is locally stable and there are two limit cycles in system (1) with the outermost $\Omega_{2}$ being stable and the innermost $\Omega_{1}$ being unstable. Furthermore, $\Omega_{2}$ intersects $L_{2}$ at two points $E_{21}\left(x_{21}, y_{21}\right)$ and $E_{24}\left(x_{24}, y_{24}\right)$ with $x_{21}<$ $x_{24}$, and $\Omega_{1}$ intersects $L_{2}$ at two points $E_{22}\left(x_{22}, y_{22}\right)$ and $E_{23}\left(x_{23}, y_{23}\right)$ with $x_{22}<x_{23}$. Similarly, based on the positions among ET, $x_{23}$, and $x_{24}$, we consider the following three cases:

$$
\begin{aligned}
& \left(C_{1}\right) \quad E T \leq x_{23}, \\
& \left(C_{2}\right) \quad E T \geq x_{24}, \\
& \left(C_{3}\right) \quad x_{23}<E T<x_{24} .
\end{aligned}
$$

These three cases are indicated in Figure 5(c). For case $\left(C_{1}\right)$, if $(1-\delta) E T \leq x_{21}$, then Is is $\mathscr{I}_{1}$ and Ps is $\mathscr{P}_{1}$. If $x_{21}<(1-$ $\delta) E T \leq x_{22}$, then we obtain Is $\mathscr{I}$ and Ps $\mathscr{P}$. From the analysis for case (i) of Lemma 4 , if $x_{22}<(1-\delta) E T<x_{P_{1}}$, then we obtain Is $\mathscr{I}_{1}$ and Ps $\mathscr{P}_{1}$. If $(1-\delta) E T \geq x_{P_{1}}$, then we have Is $\mathscr{I}$ and Ps $\mathscr{P}_{2}$. For case $\left(C_{2}\right)$, if $(1-\delta) E T<x_{P_{1}}$, then we get Is $\mathscr{I}_{1}$ and Ps $\mathscr{P}_{1}$. If $(1-\delta) E T \geq x_{P_{1}}$, then we derive Is $\mathscr{I}$ and Ps $\mathscr{P}_{2}$. For case $\left(C_{3}\right)$, if $(1-\delta) E T \leq x_{21}$, then we obtain Is $\mathscr{I}_{1}$ and Ps $\mathscr{P}_{1}$. If $x_{21}<(1-\delta) E T<x_{22}\left(\right.$ or $x_{23}<(1-\delta) E T<$ $\left.x_{24}\right)$, then Is is $\mathscr{I}$ and Ps is $\mathscr{P}$. If $x_{22} \leq(1-\delta) E T$, then $L_{3}$ must intersect $\Omega_{1}$ at two points $L C_{1}=\left((1-\delta) E T, y_{L C_{1}}\right)$ and $L C_{2}=\left((1-\delta) E T, y_{L C_{2}}\right)$ with $y_{L C_{1}}<y_{L C_{2}}$. We have Is $\mathscr{I}$ and Ps $\mathscr{P}_{3}=\left\{\left(x^{+}, y^{+}\right) \in R^{2} \mid x^{+}=(1-\delta) E T, y^{+} \in D_{3}\right\}$, where $D_{3}=\left\{\left(y_{L C_{2}},+\infty\right) \cup\left[0, y_{L C_{1}}\right)\right\}$.

In conclusion, we obtain all domains of Is and Ps of model (2) and list them in Table 1 for case $\left(A_{2}\right)$. For convenience, the same notations are used to denote cases with the same domains of Is and Ps. In particular, let (SC) be case when Is and Ps are $\mathscr{I}$ and $\mathscr{P}$; let $\left(S C_{1}\right)$ be case when Is and Ps are $\mathscr{I}_{1}$ and $\mathscr{P}_{1}$; let $\left(S C_{2}\right)$ be case when Is and Ps are $\mathscr{I}$ and $\mathscr{P}_{2}$; let $\left(S_{3}\right)$ be case when Is and Ps are $\mathscr{I}$ and $\mathscr{P}_{3}$; let $\left(S C_{4}\right)$ be case when Is and Ps are $\mathscr{I}$ and $\mathscr{P} /\left\{E^{*}\right\}$. Since the Poincaré map is well defined for cases $(S C)$ and $\left(S C_{i}\right)(i=1,2,3,4)$, and the properties of the Poincaré map as shown in Theorem 5 are all satisfied, the existence and stability of order- $k$ limit cycles can be investigated similarly by using the same methods as case $\left(A_{1}\right)$.

Based on the above analyses, the complex domains of the Poincaré map under case $\left(A_{2}\right)$ have been discussed; then the complex dynamics of system (2) can be investigated by using the same methods as those shown in Section 3. In order to avoid redundancy, we just show the results which are different from case $\left(A_{1}\right)$ in the following. 
TABLE 1: Complex domains of impulsive set and phase set of system (2) for case $\left(A_{2}\right)$.

\begin{tabular}{|c|c|c|c|c|c|}
\hline Cases & Cases & $E T$ & $(1-\delta) E T$ & Is & Ps \\
\hline$\left(S C_{1}\right)$ & (i) & $E T \geq x^{*}$ & $(1-\delta) E T<x_{P_{1}}$ & $\mathscr{I}_{1}$ & $\mathscr{P}_{1}$ \\
\hline$\left(S C_{2}\right)$ & (i) & $E T \geq x^{*}$ & $(1-\delta) E T \geq x_{P_{1}}$ & $\mathscr{I}$ & $\mathscr{P}_{2}$ \\
\hline \multirow{2}{*}{$\left(S C_{1}\right)$} & (ii) & $\left(B_{1}\right)$ & $(1-\delta) E T \leq x_{11}$ & $\mathscr{I}_{1}$ & $\mathscr{P}_{1}$ \\
\hline & (ii) & $\left(B_{2}\right)$ & $(1-\delta) E T<x_{P_{1}}$ & $\mathscr{I}_{1}$ & $\mathscr{P}_{1}$ \\
\hline$\left(S_{2}\right)$ & (ii) & $\left(B_{2}\right)$ & $(1-\delta) E T \geq x_{P_{1}}$ & $\mathscr{I}$ & $\mathscr{P}_{2}$ \\
\hline$\left(S_{4}\right)$ & (ii) & $\left(B_{3}\right)$ & $x_{11}<(1-\delta) E T<x_{12}$ & $\mathscr{I}$ & $\mathscr{P} /\left\{E^{*}\right\}$ \\
\hline$\left(S C_{1}\right)$ & (iii) & $\left(C_{1}\right)$ & $(1-\delta) E T \leq x_{21}$ & $\mathscr{I}_{1}$ & $\mathscr{P}_{1}$ \\
\hline$(S C)$ & (iii) & $\left(C_{1}\right)$ & $x_{21}<(1-\delta) E T \leq x_{22}$ & $\mathscr{I}$ & $\mathscr{P}$ \\
\hline$\left(S C_{1}\right)$ & (iii) & $\left(C_{1}\right)$ & $x_{22}<(1-\delta) E T<x_{P_{1}}$ & $\mathscr{I}_{1}$ & $\mathscr{P}_{1}$ \\
\hline$\left(\mathrm{SC}_{2}\right)$ & (iii) & $\left(C_{1}\right)$ & $x_{P_{1}} \leq(1-\delta) E T$ & $\mathscr{I}$ & $\mathscr{P}_{2}$ \\
\hline$\left(S C_{1}\right)$ & (iii) & $\left(C_{2}\right)$ & $(1-\delta) E T<x_{P_{1}}$ & $\mathscr{I}_{1}$ & $\mathscr{P}_{1}$ \\
\hline$\left(S C_{2}\right)$ & (iii) & $\left(C_{2}\right)$ & $x_{P_{1}} \leq(1-\delta) E T$ & $\mathscr{I}$ & $\mathscr{P}_{2}$ \\
\hline$\left(S C_{1}\right)$ & (iii) & $\left(C_{3}\right)$ & $(1-\delta) E T \leq x_{21}$ & $\mathscr{I}_{1}$ & $\mathscr{P}_{1}$ \\
\hline$(S C)$ & (iii) & $\left(C_{3}\right)$ & $x_{21}<(1-\delta) E T<x_{22}$ & $\mathscr{I}$ & $\mathscr{P}$ \\
\hline$\left(\mathrm{SC}_{3}\right)$ & (iii) & $\left(C_{3}\right)$ & $x_{22} \leq(1-\delta) E T$ & $\mathscr{I}$ & $\mathscr{P}_{3}$ \\
\hline
\end{tabular}

\subsection{Complex Dynamics for Case $\left(A_{2}\right)$}

Theorem 14. For case $\left(S C_{2}\right)$, if $\varphi\left(y_{\text {min }}^{P}\right) \leq y_{\text {min }}^{P}$, then there exists a stable order-1 limit cycle for system (2). If $\varphi\left(y_{\text {min }}^{P}\right) \geq$ $y_{\max }^{P}$, then there also exists a unique order-1 limit cycle for system (2). If $\varphi\left(y_{\max }^{P}\right)>y_{\max }^{P}$ and $\varphi^{2}\left(y_{\max }^{P}\right) \geq y_{\max }^{P}$, then system (2) does not have an order-k $(k \geq 3)$ limit cycle other than the stable order-1 limit cycle or order-2 limit cycle.

Proof. If $\varphi\left(y_{\min }^{P}\right)=y_{\min }^{P}$ or $\varphi\left(y_{\min }^{P}\right)=y_{\max }^{P}$, then the results are true by using the same methods as shown in Theorem 8. If $\varphi\left(y_{\text {min }}^{P}\right)<y_{\text {min }}^{P}$, then any solution initiating from $\left((1-\delta) E T, y_{0}^{+}\right)$with $y_{0}^{+} \in D_{2}$ experiences $n$ times (infinitely) impulsive effects, and $y_{n}^{+} \in\left[0, y_{\text {min }}^{P}\right]$. Further, from Theorem 5, the impulsive point series $y_{n}^{+}$starting from the interval $\left[y_{\max }^{P},+\infty\right)$ is monotonically decreasing and $y_{n}^{+}$starting from the interval $\left[0, y_{\min }^{P}\right]$ is monotonically increasing. Therefore, it is obvious that $\lim _{n \rightarrow+\infty} y_{n}^{+}=y_{f}$ with $y_{f} \in\left[0, y_{\min }^{P}\right]$; that is $\varphi\left(y_{f}\right)=y_{f}$. This implies that system (2) has a stable order-1 limit cycle.

If $\varphi\left(y_{\min }^{P}\right)>y_{\max }^{P}$, then we only need to discuss two possible cases: $\sigma \geq y_{\max }^{P}$ and $\sigma<y_{\max }^{P}$. If $\sigma \geq y_{\max }^{P}$, then any solution starting from $\left((1-\delta) E T, y_{0}^{+}\right)$with $y_{0}^{+} \in D_{2}$ experiences infinitely many pulses, and, in the light of the properties of the Poincaré map $\varphi$, there is a unique fixed point $y_{f} \in\left(y_{\max }^{P},+\infty\right)$. If $\sigma<y_{\max }^{P}$, then there exists a point $y_{C}$ such that $\varphi\left(y_{C}\right)=y_{\max }^{P}$. Moreover, any solution initiating from $\left((1-\delta) E T, y_{0}^{+}\right)$with $y_{0}^{+} \in\left[0, Y_{C}\right) \subset\left[0, y_{\min }^{P}\right]$ (or $y_{0}^{+} \epsilon$ $\left(Y_{C}^{1}, Y_{C}\right)$ and $\left.0<Y_{C}^{1}<Y_{C}\right)$ will be free from impulsive effects after one single impulsive effect. Consequently, the properties of the Poincaré map are satisfied on the interval $D_{2} /\left[0, Y_{C}\right.$ ) (or $\left.D_{2} /\left(Y_{C}^{1}, Y_{C}\right)\right)$. So system (2) has a stable order-1 limit cycle.

If $\varphi\left(y_{\max }^{P}\right)>y_{\max }^{P}$ and $\varphi^{2}\left(y_{\max }^{P}\right) \geq y_{\max }^{P}$, then, by using the same methods as in the proof of Theorem 10, system (2) does not have an order- $k(k \geq 3)$ limit cycle other than the stable order-1 limit cycle or order-2 limit cycle. This completes the proof.

Under the conditions of Theorem 14, there does not exist an order- $k(k \geq 3)$ limit cycle other than the stable order-1 limit cycle or order-2 limit cycle for system (2). By using the same methods, it is claimed that system (2) does not have an order- $k(k \geq 3)$ limit cycle for two other cases: case $\left(S C_{1}\right)$ with $\varphi\left(y_{\delta E}\right) \geq y_{\delta E}\left(\right.$ or $\left.\varphi\left(y_{\delta E}\right)<y_{\delta E}\right)$ and case $\left(S C_{3}\right)$ with $\varphi\left(y_{L C_{2}}\right) \geq$ $y_{L C_{2}}\left(\operatorname{or} \varphi\left(y_{L C_{1}}\right) \leq y_{L C_{1}}\right)$.

Remark 15. If $y_{\text {min }}^{P}<\varphi\left(y_{\text {min }}^{P}\right)<y_{\text {max }}^{P}\left(\right.$ or $y_{\text {min }}^{P}<\varphi\left(y_{\text {max }}^{P}\right)<$ $\left.y_{\max }^{P}\right)$ for case $\left(S \mathrm{~S}_{2}\right)$, then any solution with initial condition $\left((1-\delta) E T, y_{0}^{+}\right)$with $y_{0}^{+} \in D_{2}$ will experience finite pulses and then will be free from impulsive effects, and finally they will tend to the open set $\left(y_{\min }^{P}, y_{\max }^{P}\right)$. In particular, if $(1-\delta) E T \geq$ $x_{P_{1}}, E T>x^{*}$, and $y_{\min }^{P}<\varphi\left(y_{\min }^{P}\right)<y_{\max }^{P}$, then any solution with initial condition $\left((1-\delta) E T, y_{0}^{+}\right) \in \mathscr{P}_{2}$ will finally tend to the stable equilibrium $E^{*}$ after finite pulses; if $(1-\delta) E T \geq x_{P_{1}}$, $E T \geq x_{12}$, and $y_{\min }^{P}<\varphi\left(y_{\min }^{P}\right)<y_{\max }^{P}$, then any solution starting from $\left((1-\delta) E T, y_{0}^{+}\right) \in \mathscr{P}_{2}$ will finally tend to the stable limit cycle $\Omega$ after finite pulses; if $(1-\delta) E T \geq x_{P_{1}}$, $E T \geq x_{24}$, and $y_{\min }^{P}<\varphi\left(y_{\min }^{P}\right)<y_{\max }^{P}$, then any solution initiating from $\left((1-\delta) E T, y_{0}^{+}\right) \in \mathscr{P}_{2}$ will finally tend to the stable limit cycle $\Omega_{2}$ after finite pulses.

From the proof of Theorem 14, we conclude that the multistable behaviors corresponding to coexistence of the limit cycle and order- $k$ limit cycles could happen under the conditions of Remark 15. For example, an order-1 limit cycle, the limit cycle $\Omega_{1}$, and the limit cycle $\Omega_{2}$ can coexist, as shown in Figure 6. It implies that the pests and enemies may oscillate periodically along the limit cycles with different amplitudes; in other words, the final quantities at the equilibrium level for pests and enemies mainly rely on initial densities $[33,34]$. 


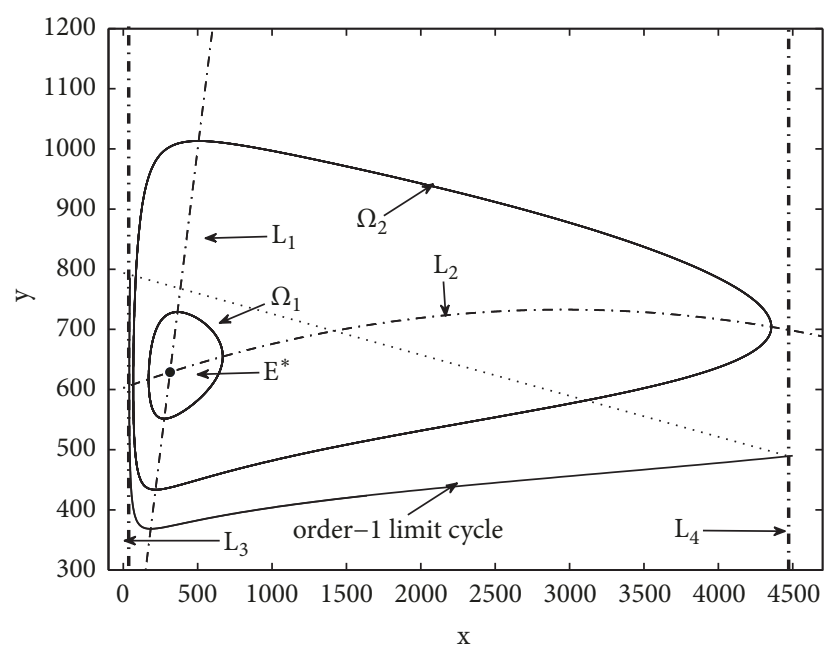

FIGURE 6: Coexistence of the limit cycles and an order-1 limit cycle with $r=0.0314425, K=10000, m=0.2123448512, A=4070.34$, $s=0.00128, h=0.5, E T=4500, \delta=0.99, \sigma=100$, and $q=0.4$.

For case $\left(S C_{4}\right)$, the Is and Ps are both located in the interior of the stable limit cycle $\Omega$; any solution initiating from Ps will meet Is and then experience impulsive effects. Furthermore, the Poincaré map has a fixed point, which corresponds to the limit cycle of system (2) (see Figure 7(a)). Compared to the results obtained from case $\left(A_{1}\right)$, the difference is that the Poincaré map in this case has complex properties. More precisely, the definition domain of the Poincaré map for system (2) has a complicated shape with discontinuity points. For example, if we fix all the parameters as those shown in Figure 7(a), then there is an order-1 limit cycle for system (2) with three discontinuity points, and then denoted by $D C_{1}, D C_{2}$, and $D C_{3}$. Moreover, it is clear that the number of discontinuity points of Poincaré map depends on the number of intersection points of spiral orbits initiating from the phase set with the impulsive set before it reaches the impulsive set. Finally, it is found that the number of discontinuity points of Poincaré map first increases and reaches its maximum value around $E^{*}$ and then decreases as the key parameter $\delta$ decreases, as shown in Figure 8. For case (SC), similar results can be obtained and we do not address them in detail (Figure 7(b)); in this case this order-1 limit cycle lies between $\Omega_{1}$ and $\Omega_{2}$.

\section{Conclusions}

Recently, mathematical models with feedback control formulated by impulsive dynamical systems [23, 24] have been applied in many fields since the problems originating from these areas can be modelled very well when human actions are taken for real word applications [14-21], rather than fixed pulsed models. Nevertheless, a very important and well-known biological model named the Holling-Tanner model, also known as the model of R. M. May, has never been studied when incorporating state-dependent feedback control because the system has very complex dynamics. It is known that mathematical analysis of the Holling-Tanner model with impulsive control not only possesses significant theoretical implications, but also indicates biological meanings. Therefore, the paper proposes a novel Holling-Tanner model with impulsive feedback control. The objective is to provide a comprehensive analysis for global dynamics of system (2) and show how state-dependent feedback control strategy affects the dynamics.

The Poincaré map is first constructed in the phase sets considering phase portraits of model (1). Based on the positions between the threshold $E T$ and the equilibrium $E^{*}$, we consider two cases: $\left(A_{1}\right) E T<x^{*}$ and $\left(A_{2}\right) E T \geq x^{*}$. For case $\left(A_{1}\right)$, the main properties of the Poincaré map which play important roles in the proof of the existence of limit cycles are firstly investigated. Then the existence and stability of the boundary order-1 limit cycle are studied, and it is shown that this limit cycle is always unstable. It means that the pest itself can not be maintained below the threshold when chemical control only is applied, and biological control such as predators should be introduced for successful pest control. Subsequently, the conditions for the existence and stability of the order- 1 limit cycle are provided by employing the fixed point theorems and analogue of Poincaré criterion, revealing that the pests and enemies will oscillate periodically below ET. Moreover, we have also studied the existence of order $-k(k \geq 2)$ limit cycle, and the conditions for the existence of an order-3 limit cycle are provided, which confirms that there exists limit cycles of any period for system (2) [31, 32]. After that, numerical investigations with respect to control parameters are carried out to substantiate our results, and these results further show that there exists rich and complex dynamics for system (2) which are consistent with the theoretical results.

For case $\left(A_{2}\right)$, the complex domains of the impulsive set and phase set are given for system (2) and the results are listed in Table 1. In order to avoid redundancy, the results that are different from case $\left(A_{1}\right)$ are shown. In particular, under certain conditions there does not exist an order $-k(k \geq 3)$ limit cycle other than the order-1 limit cycle or order-2 limit cycle for system (2), and multistable behaviors are observed which indicate that the final states of the pest and natural enemy populations mainly depend on their initial densities as well as on their ratios. Once the impulsive set and phase set are both located in the interior of the stable limit cycle $\Omega$, it is found that the definition domain of the Poincaré map has a complicated shape with discontinuity points, and it is concluded that the number of discontinuity points first increases and reaches its maximum value around $E^{*}$ and then decreases as the control parameter $\delta$ decreases.

Compared to the previous studies with impulsive feedback control $[17,18,20]$, we list the differences: (1) in references $[17,18,20]$, the impulsive and phase sets are often defined for some special cases, while in this paper all possible cases are discussed and listed in Table 1; (2) in previous studies, the boundary order-1 limit cycle could be stable for some parameter sets. However, in this paper, the boundary order-1 limit cycle is unstable over the whole parameter space due to the complexity of system (1), suggesting that chemical control on its own cannot reach the target of successful pest control; (3) Theorem 14 provided conditions 


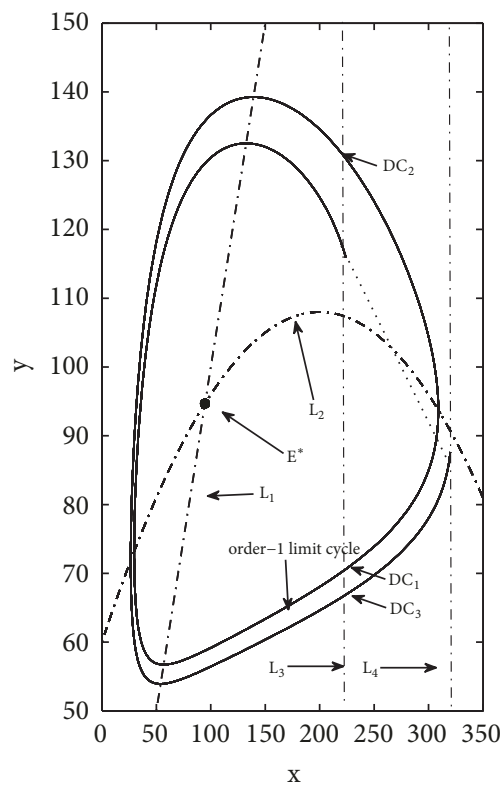

(a)

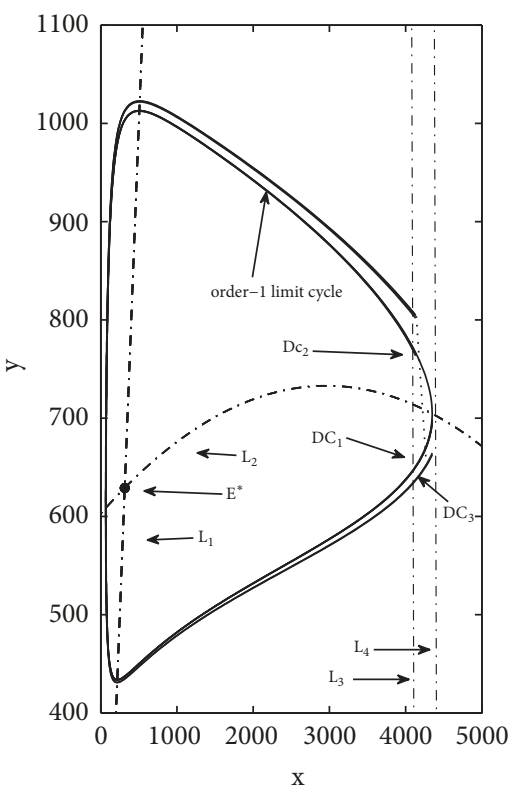

(b)

Figure 7: Discontinuity of Poincaré map. (a) For case $\left(S C_{4}\right)$ of system (2) with $r=0.3, K=500, m=0.5, A=100, s=0.03, h=1, E T=3200$, $\delta=0.3, \sigma=2$, and $q=0.3$. (b) For case (SC) of system (2) with $r=0.0314425, K=10000, m=0.2123448512, A=4070.34, s=0.00128$, $h=0.5, E T=4350, \delta=0.05, \sigma=1$, and $q=0.15 . D C_{1}, D C_{2}$, and $D C_{3}$ represent the discontinuity points.

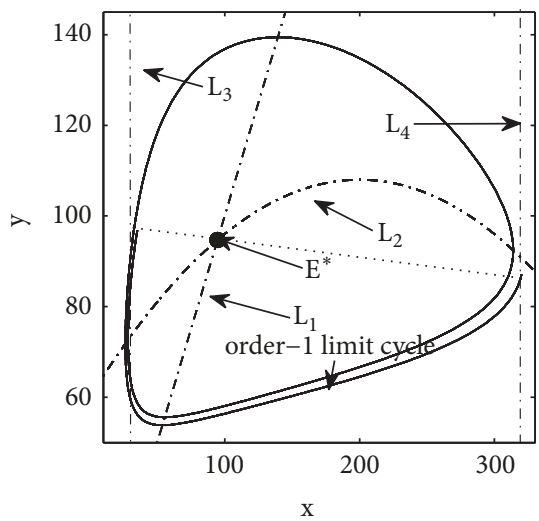

(a)

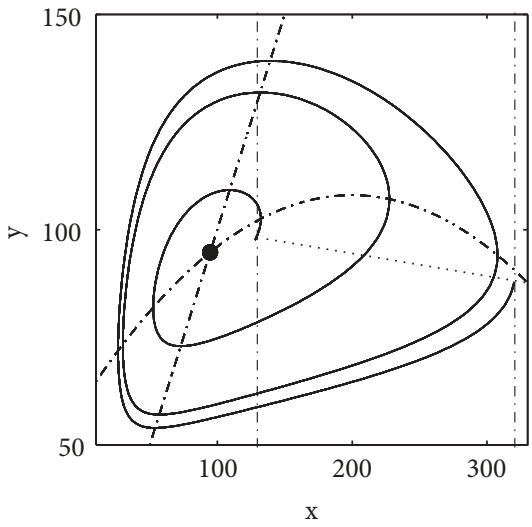

(d)

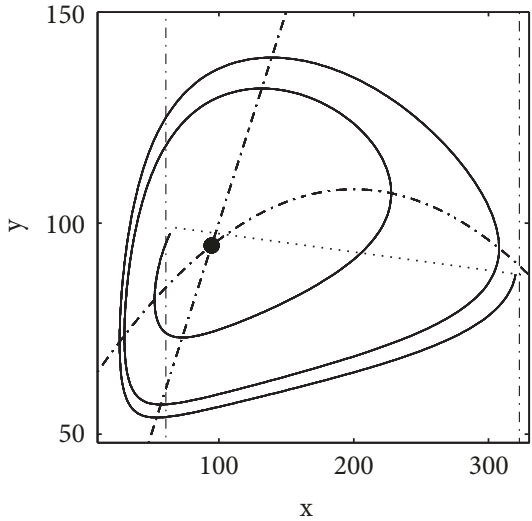

(b)

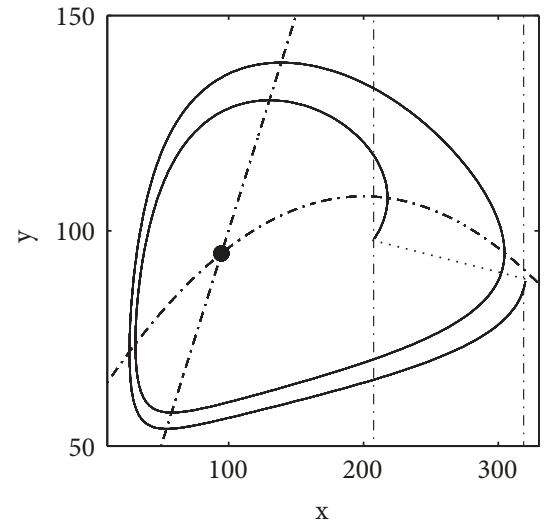

(e)

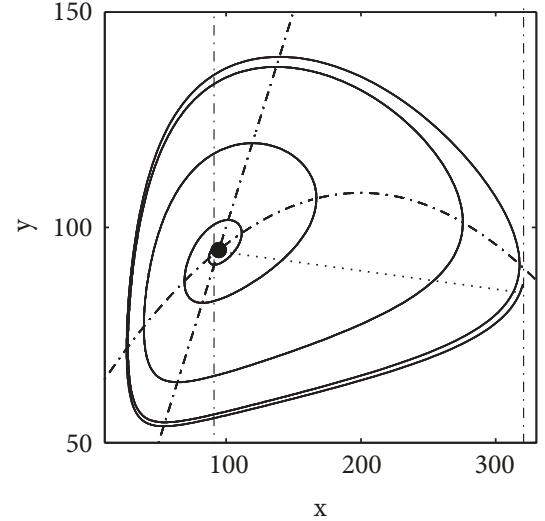

(c)

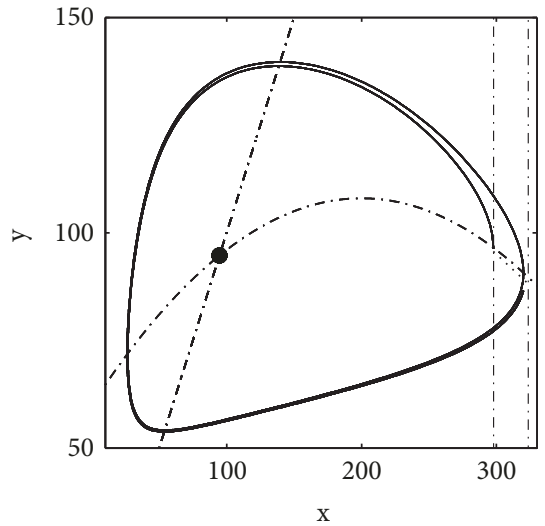

(f)

Figure 8: Number of discontinuity points of Poincaré map for case $\left(S C_{4}\right)$ with $r=0.3, K=500, m=0.5, A=100, s=0.03, h=1, E T=320$, $\sigma=2$, and $q=0.3$. (a) $\delta=0.89$; (b) $\delta=0.8$; (c) $\delta=0.7$; (d) $\delta=0.6$; (e) $\delta=0.35$, and (f) $\delta=0.07$. 
for the existence of the multistable behaviors; that is to say, the order-1 limit cycle, the limit cycle $\Omega_{1}$, and the limit cycle $\Omega_{2}$ can coexist (Figure 6); this is much more complex compared to the results as shown in previous studies $[17,18,20]$; $(4)$ the existence of order-3 limit cycle has been proved rather than order-1 or order-2 limit cycle; (5) the discontinuity points of the Poincaré map have also been addressed. In conclusion, all the results and methods presented in this paper enrich and improve the previous studies related to state-dependent feedback control.

There are studies about the development and extinction of predators affected by resource limitations $[13,14]$. How could we incorporate the effect of resource limitation into the prey-predator system (2) and assess how resource limitation affects the dynamics of the system (2) and further affects the outcome of pest control? These topics will be studied in the near future. Moreover, an ideal control strategy is that integrated pest management (IPM) strategies should be implemented once the density of the pest population grows and exceeds the economic threshold (ET), while control tactics are suspended once the density of the pest population falls below the ET again. To address these, complex nonsmooth model or Filippov system needs to be developed and investigated within an IPM context $[35,36]$, which we plan to do in the near future.

\section{Data Availability}

No data were used to support this study.

\section{Conflicts of Interest}

The authors declare that there are no conflicts of interest regarding the publication of this paper.

\section{Acknowledgments}

This work was supported by the Chongqing Municipal Education Commission (KJ1600522), Basic and Advanced Research Project of Chongqing (cstc2017jcyjAX0131 and cstc2016jcyjA0412), and National Natural Science Foundation of China (NSFC 11761031 and 11801047).

\section{References}

[1] R. M. May, "Limit cycles in predator-prey communities," Science, vol. 177, no. 4052, pp. 900-902, 1972.

[2] R. M. May, Stabilityand Complexityin Model Ecosystems, Princeton University Press, Princeton, NJ, USA, 1973.

[3] E. Sáez and E. González, "Dynamics of a predator-prey model," SIAM Journal on Applied Mathematics, vol. 59, no. 5, pp. 18671878, 1999.

[4] J. T. Tanner, "The stability and the intrinsic growth rates of prey and predator populations," Ecology, vol. 56, no. 4, pp. 855-867, 1975.

[5] D. J. Wollkind, J. B. Collings, and J. A. Logan, "Metastability in a temperature-dependent model system for predator-prey mite outbreak interactions on fruit trees," Bulletin of Mathematical Biology, vol. 50, no. 4, pp. 379-409, 1988.
[6] S. B. Hsu and T. W. Huang, "Global stability for a class of predator-prey systems," SIAM Journal on Applied Mathematics, vol. 55, no. 3, pp. 763-783, 1995.

[7] A. Gasull, R. E. Kooij, and J. Torregrosa, "Limit cycles in the Holling-Tanner model," Publicacions Matematiques, vol. 41, no. 1, pp. 149-167, 1997.

[8] S. Tang and R. A. Cheke, "Models for integrated pest control and their biological implications," Mathematical Biosciences, vol. 215, no. 1, pp. 115-125, 2008.

[9] J. C. Van Lenteren, Integrated Pest Management in Protected Crops Integrated Pest Management, Chapman and Hall, London, UK, 1995.

[10] J. C. Van Lenteren and J. Woets, "Biological and integrated pest control in greenhouses," Annual Review of Entomology, vol. 33, no. 1, pp. 239-250, 1988.

[11] X. Liu and L. Chen, "Complex dynamics of Holling type II Lotka-Volterra predator-prey system with impulsive perturbations on the predator," Chaos, Solitons \& Fractals, vol. 16, no. 2, pp. 311-320, 2003.

[12] B. Liu, Z. Teng, and L. Chen, "Analysis of a predator-prey model with Holling II functional response concerning impulsive control strategy," Journal of Computational and Applied Mathematics, vol. 193, no. 1, pp. 347-362, 2006.

[13] W. Qin, S. Tang, and R. A. Cheke, "The effects of resource limitation on a predator-prey model with control measures as nonlinear pulses," Mathematical Problems in Engineering, vol. 2014, Article ID 450935, 13 pages, 2014.

[14] J. Yang and S. Tang, "Holling type II predator-prey model with nonlinear pulse as state-dependent feedback control," Journal of Computational and Applied Mathematics, vol. 291, pp. 225-241, 2016.

[15] G. Tang, J. Yang, and S. Tang, "Qualitative analysis of a quadratic integrate-and-fire neuron model with state-dependent feedback control," Discrete Dynamics in Nature and Society, vol. 2015, Art. ID 836402, 12 pages, 2015.

[16] M. Z. Huang, J. X. Li, X. Y. Song, and H. J. Guo, "Modeling impulsive injections of insulin: towards artificial pancreas," SIAM Journal on Applied Mathematics, vol. 72, no. 5, pp. 15241548, 2012.

[17] G. Jiang, Q. Lu, and L. Qian, "Complex dynamics of a Holling type II prey-predator system with state feedback control," Chaos, Solitons \& Fractals, vol. 31, no. 2, pp. 448-461, 2007.

[18] B. Liu, Y. Tian, and B. Kang, "Dynamics on a Holling II predator-prey model with state-dependent impulsive control," International Journal of Biomathematics, vol. 5 , no. 3, Article ID 1260006, pp. 93-110, 2012.

[19] Y. Lv, R. Yuan, and Y. Pei, “Two types of predator-prey models with harvesting: non-smooth and non-continuous," Journal of Computational and Applied Mathematics, vol. 250, pp. 122-142, 2013.

[20] S.-Y. Tang, B. Tang, A.-L. Wang, and Y.-N. Xiao, "Holling II predator-prey impulsive semi-dynamic model with complex Poincaré map," Nonlinear Dynamics, vol. 81, no. 3, pp. 15751596, 2015.

[21] S. Y. Tang, W. H. Pang, R. A. Cheke, and J. H. Wu, "Global dynamics of a state-dependent feedback control system," Advances in Difference Equations, vol. 2015, article 322, 2015.

[22] L. Nie, Z. Teng, L. Hu, and J. Peng, "Qualitative analysis of a modified Leslie-Gower and Holling-type II predatorprey model with state dependent impulsive effects," Nonlinear Analysis: Real World Applications, vol. 11, no. 3, pp. 1364-1373, 2010. 
[23] P. S. Simeonov and D. D. Bainov, "Orbital stability of periodic solutions of autonomous systems with impulse effect," International Journal of Systems Science, vol. 19, no. 12, pp. 2561-2585, 1988.

[24] D. Bainov and P. Simeonov, Impulsive Differential Equations: Periodic Solutions and Applications, vol. 66, Longman Scientific and Technical, New York, NY, USA, 1993.

[25] K. Ciesielski, "On semicontinuity in impulsive dynamical systems," Bulletin of the Polish Academy of Sciences. Mathematics, vol. 52, no. 1, pp. 71-80, 2004.

[26] K. Ciesielski, "On stability in impulsive dynamical systems," Bulletin of the Polish Academy of Sciences. Mathematics, vol. 52, no. 1, pp. 81-91, 2004.

[27] K. Ciesielski, "On time reparametrizations and isomorphisms of impulsive dynamical systems," Annales Polonici Mathematici, vol. 84, no. 1, pp. 1-25, 2004.

[28] S. K. Kaul, "On impulsive semidynamical systems," Journal of Mathematical Analysis and Applications, vol. 150, no. 1, pp. 120128, 1990.

[29] S. Kaul, "On impulsive semidynamical systems III: Lyapunov stability," Recent Trends in Differential Equations, vol. 1, pp. 335345, 1992.

[30] A. Matveev and A. Savkin, Qualitative Theory of Hybrid Dynamical Systems, Cambridge University Press, Cambridge, UK, 2000.

[31] R. L. Devaney, An Introduction to Chaotic Dynamical Systems, Addison-Wesley, Redwood City, Calif, USA, 1989.

[32] T. Y. Li and J. A. Yorke, "Period three implies chaos," The American Mathematical Monthly, vol. 82, no. 10, pp. 985-992, 1975.

[33] G. Foster and A. Kelly, "Initial density of glasshouse whitefly (Trialeurodes vaporariorum (Westwood), Hemiptera) in relation to the success of suppression by Encarsia formosa Gahan (Hymenoptera) on glasshouse tomatoes," Horticultural Research, vol. 18, pp. 55-62, 1978.

[34] W. A. Jones, S. M. Greenberg, and B. Legaspi Jr., “The effect of varying Bemisia argentifolii and Eretmocerus mundus ratios on parasitism," BioControl, vol. 44, no. 1, pp. 13-28, 1999.

[35] J. Yang, S. Tang, and R. A. Cheke, "Global stability and sliding bifurcations of a non-smooth Gause predator-prey system," Applied Mathematics and Computation, vol. 224, pp. 9-20, 2013.

[36] M. di Bernardo, C. J. Budd, A. R. Champneys et al., "Bifurcations in nonsmooth dynamical systems," SIAM Review, vol. 50, no. 4, pp. 629-701, 2008. 


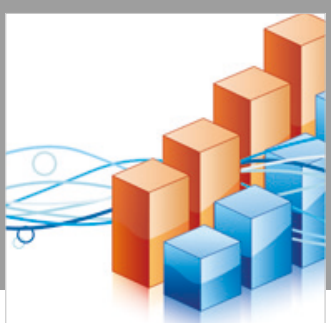

Advances in

Operations Research

\section{-n-m}
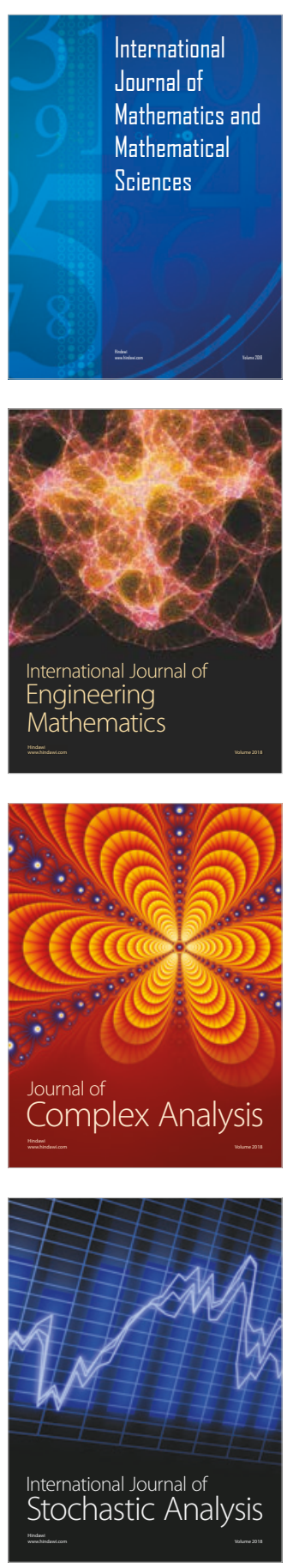
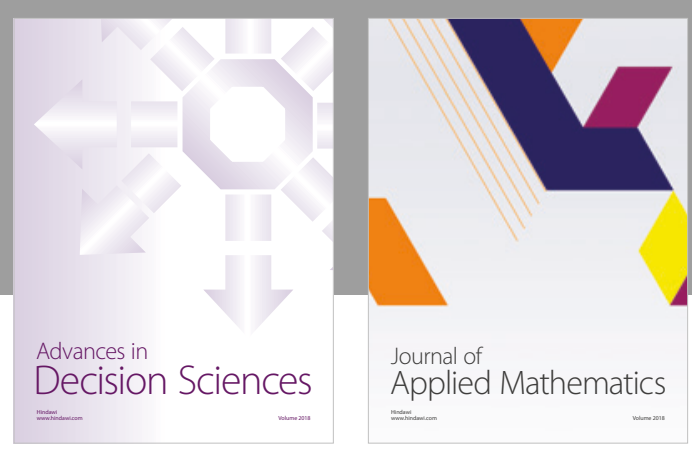

Journal of

Applied Mathematics
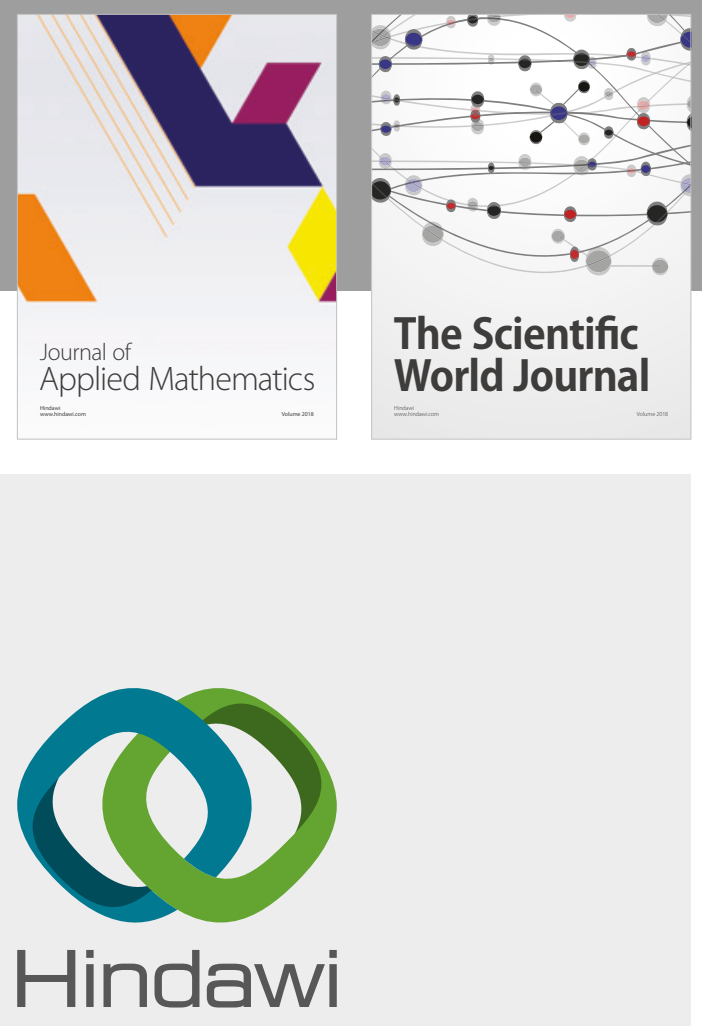

Submit your manuscripts at

www.hindawi.com

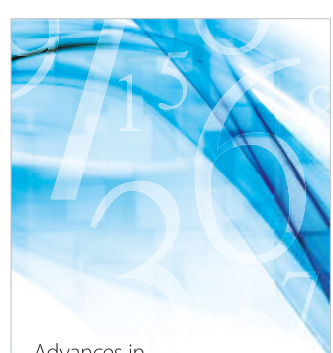

Advances in
Numerical Analysis
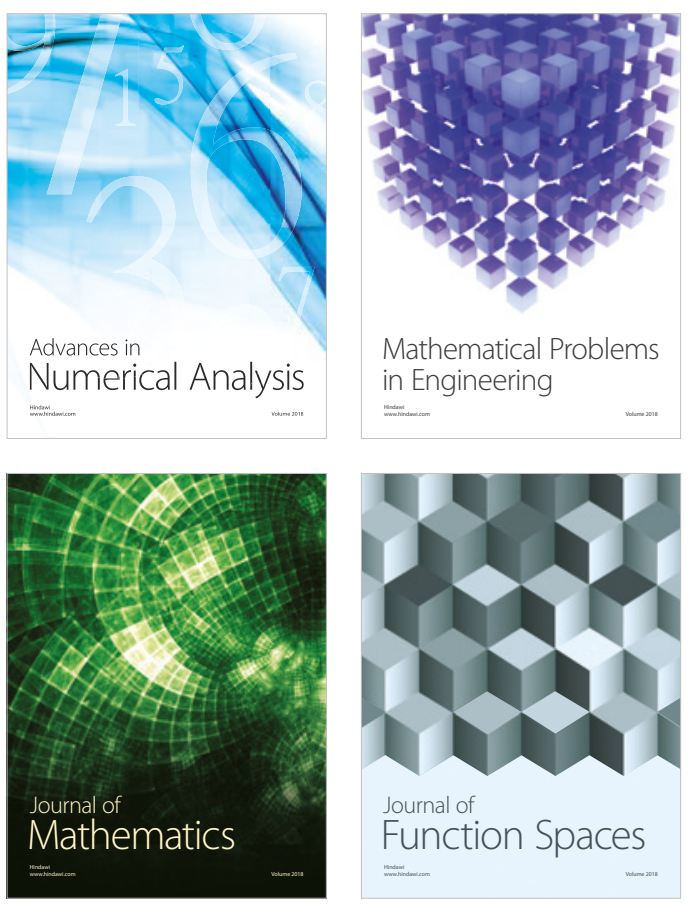

Mathematical Problems in Engineering

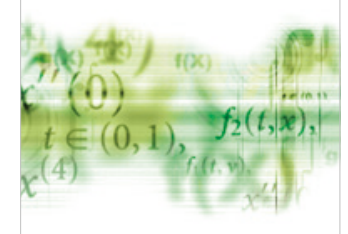

International Journal of

Differential Equations

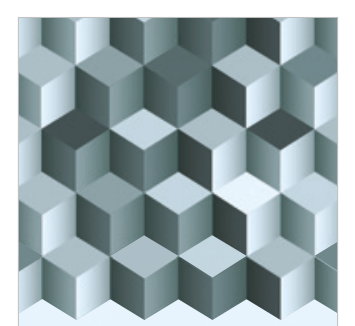

Journal of

Function Spaces
The Scientific

World Journal

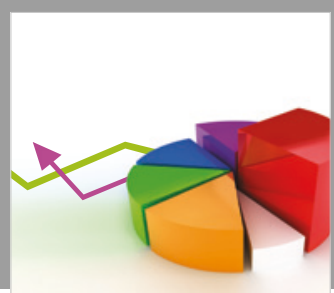

Journal of

Probability and Statistics
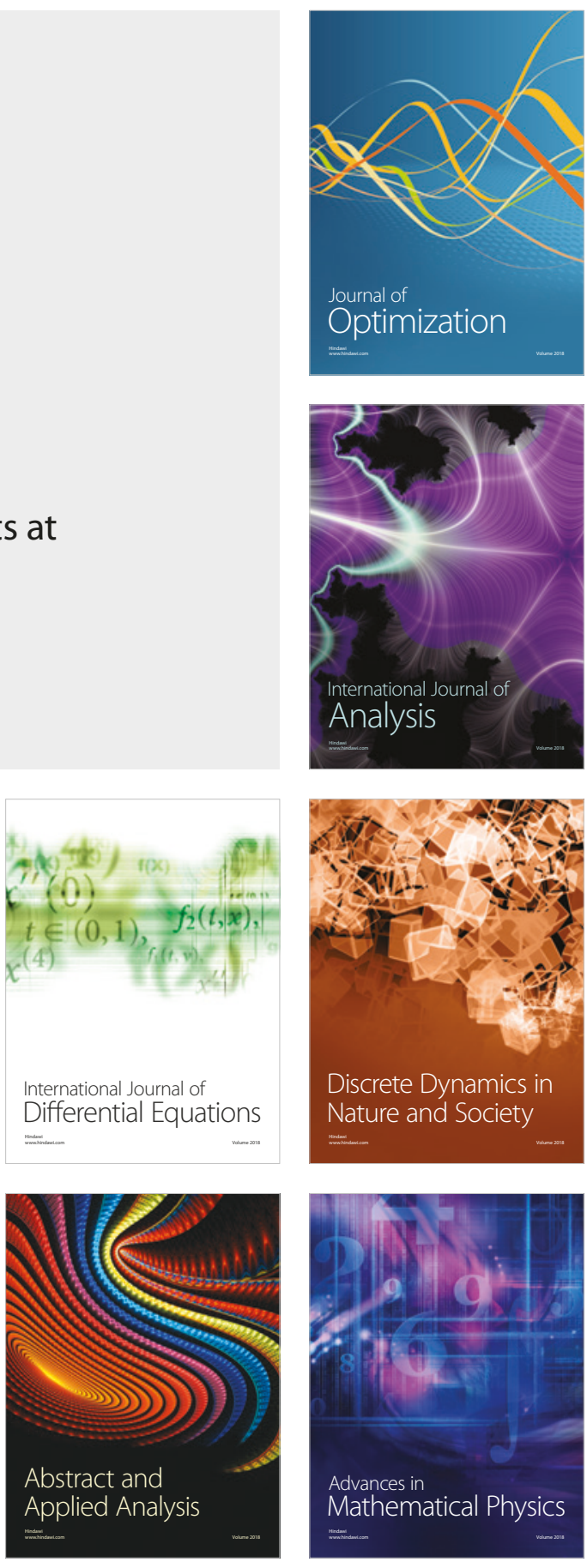